\title{
ARTICLE \\ CCR2 mediates increased susceptibility to post-H1N1 bacterial pneumonia by limiting dendritic cell induction of
} IL-17

Stephen J. Gurczynski ${ }^{1}$, Niket Nathani ${ }^{1}$, Helen I. Warheit-Niemi ${ }^{2}$, Elissa M. Hult ${ }^{2}$, Amy Podsiad ${ }^{1}$, Jane Deng ${ }^{1,3}$, Rachel L. Zemans ${ }^{1}$, Urvashi Bhan ${ }^{1,4}$ and Bethany B. Moore ${ }^{1,2}$

Post influenza bacterial pneumonia is associated with significant mortality and morbidity. Dendritic cells (DCs) play a crucial role in host defense against bacterial pneumonia, but their contribution to post influenza-susceptibility to secondary bacterial pneumonia is incompletely understood. WT and CCR2 $2^{-1-}$ mice were infected with 100 plaque forming units (pfu) H1N1 intranasally alone or were challenged on day 5 with $7 \times 10^{7}$ colony forming units (cfu) methicillin-resistant Staphylococcus aureus intratracheally. WT mice express abundant CCL2 mRNA and protein post-H1N1 alone or dual infection. CCR2 ${ }^{-1-}$ mice had significantly higher survival as compared to WT mice, associated with significantly improved bacterial clearance at 24 and $48 \mathrm{~h}$ (10-fold and 14-fold, respectively) post bacterial challenge. There was robust upregulation of IL-23 and IL-17 as well as downregulation of IL-27 expression in CCR2 ${ }^{-1-}$ mice following sequential infection as compared to WT mice, which was also associated with significantly greater accumulation of $\mathrm{CD}_{103^{+}} \mathrm{DC}$. Finally, WT mice treated with a CCR2 inhibitor showed improved bacterial clearance in association with similar cytokine profiles as CCR2 ${ }^{-1-}$ mice. Thus, CCR2 significantly contributes to increased susceptibility to bacterial infection after influenza pneumonia likely via altered dendritic cell responses and thus, CCR2 antagonism represents a potential therapeutic strategy.

Mucosal Immunology (2019) 12:518-530; https://doi.org/10.1038/s41385-018-0106-4

\section{INTRODUCTION}

Seasonal influenza A virus results in significant morbidity and mortality every year. In the United States alone, the Centers for Disease Control and Prevention estimate that seasonal influenza has been responsible for over 35 million cases, up to 710,000 hospitalizations and 56,000 deaths since 2010 (https://www.cdc. gov/flu/about/disease/2015-16.htm). In 2009, mortality reached pandemic levels, and the 2017 influenza season was also quite severe. ${ }^{1}$ Antigenic drift and shift of hemagglutinin and neuraminidase are mechanisms for influenza to evade protective memory immune responses from prior exposure or vaccination ${ }^{2}$ and thus, innate immunity is paramount for host protection.

Mortality from influenza is often due to secondary bacterial pneumonia with pathogens such as Streptococcus pneumoniae and Staphylococcus aureus as well as from the resulting inflammatory response as opposed to overwhelming viral replication. $^{3}$ In the 2009 H1N1 epidemic, infection with methicillinresistant Staphylococcus aureus (MRSA) was a major bacterial complication. ${ }^{4}$ Mounting evidence suggests that influenza modulates the innate immune response. ${ }^{2,5}$ Recruitment of key innate immune cells, including monocyte-derived dendritic cells (DC) and macrophages to the lung is mediated via CC chemokine receptor 2 (CCR2). CCR2's interaction with its high-affinity ligand, CCL2, is an essential mechanism for monocyte cell trafficking from bone marrow to sites of inflammation. ${ }^{6}$ Early cytokine/chemokine dysregulation, including elevated CCL2 protein levels in bronchoalveolar lavage (BAL) fluid are found in mouse models of influenza pneumonia and have been associated with the accumulation of CCR2 ${ }^{+}$monocytes, macrophages, and NK cells., ${ }^{7,8}$ Additionaly, $\mathrm{CCR}^{+}$cells have been suggested to contribute to lung injury and mortality in murine single infection influenza pneumonia models. ${ }^{8-10}$ However, $\mathrm{CCR}^{+}$cells are also believed to be important for clearance of bacterial pathogens in many settings. ${ }^{11-14}$ Thus, it is difficult to predict how CCR2 function may ultimately impact disease outcomes in the setting of secondary bacterial infection post-influenza.

Previous studies have shown the importance of $\mathrm{IL}-17$ in promoting bacterial clearance post-influenza and IL-17 is also important for clearance of MRSA. ${ }^{15-18}$ Antigen presenting cells (APCs) in the lung produce IL-1 $\beta$ and IL-23 in response to various inflammatory stimuli and these cytokines activate STAT3mediated transcription of IL-17 and IL-22, promoting differentiation of $\mathrm{T}$ cells, neutrophil recruitment, and antimicrobial peptide production. ${ }^{1-21}$ In mouse models of influenza pneumonia, expression of IL-1 $\beta$ and IL-23 as well as the downstream cytokines $\mathrm{IL}-17$ and IL-22 are reduced. ${ }^{16,22}$ Furthermore, infection with influenza can directly suppress IL-17 production through upregulation of miR-155. ${ }^{23}$ As a result, initial infection by influenza virus exacerbates bacterial pneumonia. ${ }^{16,24,25}$ The exact mechanism of how CCR2 ${ }^{+}$leukocytes and IL-17 intersect to control immunity in

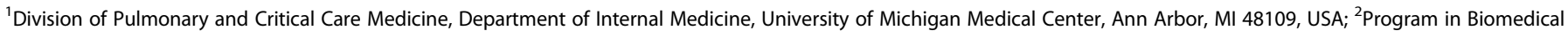
Sciences, University of Michigan, Ann Arbor, MI 48109, USA; ${ }^{3}$ Veteran's Affairs Administration, Ann Arbor, MI 48109, USA and ${ }^{4}$ St. Joseph Hospital, Ypsilanti, MI 48197, USA Correspondence: Bethany B. Moore (bmoore@umich.edu) 
the setting of post-influenza pneumonia is not known. However, we previously reported that loss-of-CCR2 ${ }^{+}$monocyte recruitment enhanced IL-17 responses to $\mathrm{y}$-herpesviruses, ${ }^{26}$ and thus we hypothesized that loss-of-CCR2 signaling would promote IL-17 responses and improve bacterial clearance following influenza infection.

With this study, we identify CCR2 as a critical inhibitor of host immune responses post sequential pneumonia. Mice deficient in CCR2 $\left(\mathrm{CCR}^{-1-}\right)$ had significantly improved mortality and improved bacterial clearance from the lung in comparison to WT mice. Additionally, CCR2 ${ }^{-1-}$ mice exhibited decreased recruitment of inflammatory monocytes to the lungs in response to both $\mathrm{H} 1 \mathrm{~N} 1$ alone and H1N1/MRSA dual infection as compared to WT mice; however, accumulation of CD103+ dendritic cells was significantly enhanced in $\mathrm{CCR}^{-1-}$ mice. Furthermore, CD11c+ DCs from $\mathrm{CCR}^{-1-}$ mice had increased expression of pro-Th17 genes which resulted in increased numbers of IL-17 producing lymphocytes and neutrophils in the lungs following infection. Antagonizing IL-17 partially abrogated the protection seen in $\mathrm{CCR}^{-1-}$ mice. Finally, treatment of infected WT mice with a CCR2 antagonist significantly improved lung bacterial clearance as compared to infected mice treated with saline. Together, these data suggest that neutralization of CCR2 following influenza infection could be efficacious in treating post-influenza secondary bacterial pneumonia.

\section{MATERIALS AND METHODS}

Mouse, virus, and bacteria strains

Six- to eight-wk-old C57BL/6 mice were purchased from The Jackson Laboratory (Bar Harbor, ME). The animals were housed in specific pathogen-free conditions within the University of Michigan Animal Care Facility (Ann Arbor, MI) until the day of death or euthanasia. CCR2 ${ }^{-1-}$ mice were bred at the University of Michigan and were age and sex matched to WT controls. All animal experiments were performed in accordance with National Institutes of Health policies on the human care and use of laboratory animals and were approved by the Institutional Animal Committee on Use and Care (IACUC) at the University of Michigan.

All experiments used a mouse-adapted influenza $A$ virus (strain $A$ / PR8/34: H1N1 isotype, ATCC) which was inoculated intranasally (i.n.) in a volume of $20 \mu \mathrm{l}$ containing 100 plaque forming units (pfu).

MRSA strain USA 300 obtained from the Network of Antimicrobial Resistance in Staphylococcus aureus (NARSA), was grown in Nutrient broth (Difco, Detroit, MI) overnight at $37^{\circ} \mathrm{C}$ with constant shaking and quantitated by measuring the amount of absorbance at $600 \mathrm{~nm}$ compared to a predetermined standard curve. Bacteria were diluted to the desired concentration for oropharyngeal inoculation. Mice were anesthetized with ketamine and xylazine by the intraperitoneal route. For oropharyngeal instillations, $30 \mu \mathrm{l}$ was deposited by pipet tip to the back of the throat while the tongue was pulled forward. An aliquot of the inoculated MRSA suspension was serially diluted onto nutrient agar plates to determine actual dose of inoculated bacteria.

Antibody purification and administration

We isolated anti-mouse IL-17 antibody from rabbit serum using Pierce Biotechnology Protein A columns and the Thermo Scientific manufacturer's protocol. A Nanodrop 2000 was used to measure concentration by absorbance at $280 \mathrm{~nm}$. The specified dose of antibody was diluted with PBS to $100 \mu \mathrm{L}$ and injected i.p. $6 \mathrm{~h}$ before MRSA inoculation.

CCR2 antagonist administration

A small molecule inhibitor of CCR2 identified as PF-04178903 of molecular formula C25 H37 F3 N4 O3 was obtained from Pfizer,
Inc. A 50- $\mu$ g subcutaneous injection of the inhibitor in a volume of $100 \mu \mathrm{L}$ was administered at the specified time points.

Whole-lung homogenization for CFU and cytokine analysis At designated time-points, mice were killed by inhalation of $\mathrm{CO}_{2}$. The lungs were perfused with $1 \mathrm{~mL}$ PBS/5 mM EDTA. Lung tissue was subsequently homogenized in $1 \mathrm{~mL}$ PBS with the addition of complete protease inhibitor (Roche, Branford, CT). CXCL1 and CCL2 ELISA assays were performed using Duoset ELISA kits (R\&D Systems, Minneapolis, MN) following the manufacturer protocol. Determination of lung bacterial load was measured via serial dilution of whole-lung homogenates on nutrient agar plates.

Total lung leukocyte preparation and alveolar epithelial cell isolation

At designated time-points, mice were killed by inhalation of $\mathrm{CO}_{2}$ and lungs were perfused with 3-5 mL PBS/EDTA. Lungs were minced with scissors to a fine slurry in $15 \mathrm{~mL}$ lung digestion buffer [DMEM, $10 \%$ fetal calf serum, $1 \mathrm{mg} / \mathrm{mL}$ collagenase $A$ (Boehringer Mannheim Biochemical) with the addition of $30 \mu \mathrm{g} / \mathrm{ml}$ DNAse (Sigma, St. Louis, MO)]. Lung slurries were enzymatically digested and centrifuged through a $20 \%$ Percoll gradient to enrich for leukocytes prior to further analyses. Cell counts, and viability were determined using trypan blue exclusion counting on a hemacytometer.

Alveolar epithelial cells were isolated by dispase digestion of whole lungs after casting the airways in low melt agarose as previously described. ${ }^{27}$ Following dispase digestion, hematopoietic cells were negatively selected with CD45 magnetic beads and remaining cells were plated overnight in DMEM $+10 \%$ FBS to adhere fibroblasts and macrophages. Non-adherent cells containing epithelial cells were then plated on fibronectin coated plates and adhered for $72 \mathrm{~h}$ before use.

Bronchoalveolar lavage, neutrophil isolation, and ex vivo neutrophil function assays

Alveolar cells were collected by repeated lavage of the lungs using a total of $20 \mathrm{ml}$ DMEM supplemented with $10 \% \mathrm{FBS}$, pen/strep, and L-glutamine via direct cannulation of the trachea with tubing attached to 2 syringes (one to instill media and one to collect media) connected by a 3-way stopcock. For some experiments, neutrophils were isolated following BAL via Ficoll/Histopaque gradient purification. Subsequent ex vivo determination of bactericidal activity was assayed via addition of MTT as previously described. ${ }^{28}$

CD11c cell isolation and isolation of RNA for qRT-PCR

After total lung leukocyte preparation was performed, cells were resuspended up to $10^{7}$ cells in $1 \mathrm{~mL}$ MACS buffer (Miltenyi Biotec, San Diego, $(A)$, incubated with $100 \mu \mathrm{L}$ of $\mathrm{CD} 11 \mathrm{c}$ magnetic microbeads (Miltenyi Biotec, San Diego, CA) and isolated via a quadroMACS magnet in a LS column (Miltenyi Biotec, San Diego, CA). Cells were then counted via a hemacytometer then cytokine measurements were performed.

Total RNA was obtained from isolated cells, or whole-lung tissue via Trizol extraction following the manufacturer's protocol. RNA concentration was normalized between samples and qRT-PCR was performed on an ABI StepOnePlus real-time thermocycler (Thermo Fisher, Waltham, MA) using a TaqMan ${ }^{\circledR}$ RNA-to-C ${ }^{\mathrm{TM}}{ }^{1}$ 1-Step Kit (Thermo Fisher, Waltham, MA) following the manufacturer's protocol. All Primers and probes used in this study are listed in Table 1.

Cell marker staining and flow cytometry with tSNE analysis After total lung leukocyte preparation was performed, non-specific Fc binding was blocked with a CD16/32 antibody. Subsequently, primary antibodies were added to cell samples and incubated for $30 \mathrm{~min}$ in the dark at $4{ }^{\circ} \mathrm{C}$. Primary antibodies used were 


\begin{tabular}{|c|c|c|}
\hline Gene name & & Primer sequence \\
\hline \multirow[t]{3}{*}{ IL-17A } & Forward & 5'-CCGCAATGAAGACCCTGATAG-3' \\
\hline & Reverse & 5'-GCTTTCCCTCCGCATTGA-3' \\
\hline & Probe & 5'-GGGAAGCTCAGTGCCGCCAG-3' \\
\hline \multirow[t]{3}{*}{ CXCL1 } & Forward & 5'-GCGCCTATCGCCAATGAG-3' \\
\hline & Reverse & 5'-GCAACACCTTCAAGCTCTGGAT-3' \\
\hline & Probe & 5'-TGCCTGCAGACCATGGCTGGG-3' \\
\hline \multirow[t]{3}{*}{ CXCL2 } & Forward & 5'-CCTGCCAAGGGTTGACTTCA-3' \\
\hline & Reverse & 5'-CCTTGAGAGTGGCTATGACTTCTG-3' \\
\hline & Probe & 5'-CGCCCCCAGGACCCCACTG-3' \\
\hline \multirow[t]{3}{*}{ IL-6 } & Forward & 5'-GACTTCCATCCAGTTGCCTTCT-3' \\
\hline & Reverse & 5'-CTGTTGGGAGTGGTATCCTCTGT-3' \\
\hline & Probe & 5'-TGACAACCACGGCCTTCCCTA-3' \\
\hline \multirow[t]{3}{*}{ IL-23 } & Forward & 5'-CTCCCTACTAGGACTCAGCCAACT-3' \\
\hline & Reverse & 5'-ACTCAGGCTGGGCATCTGTT-3' \\
\hline & Probe & 5'-AGCCAGAGGATCACCCCCGGG-3' \\
\hline \multirow[t]{3}{*}{ IL-27 } & Forward & 5'-AGGGAATTCACAGTCAGCCT-3' \\
\hline & Reverse & 5'-AGATTCAGCAAAGCTGTGGA-3' \\
\hline & Probe & 5'-CCTTGCCAGGAAGCTGCTCTCTG-3' \\
\hline \multirow[t]{3}{*}{ IL-1 $\beta$} & Forward & 5'-GAGCCCATCCTCTGTGACTCA-3' \\
\hline & Reverse & 5'-GTTGTTCATCTCGGAGCCTGTAG-3' \\
\hline & Probe & 5'-AACCTGCTGGTGTGTGACGTTCCCA-3' \\
\hline \multirow[t]{3}{*}{ IL-17r } & Forward & 5'-CTCCGACTCAGAGAACCACA-3' \\
\hline & Reverse & 5'-AGCTCGCTGATGGAATTCTT-3' \\
\hline & Probe & 5'-AACAAATATTTGGGCCCAGGCAA-3' \\
\hline
\end{tabular}

anti-CD45, CD11b, CD4, IL-17A, MHCII (I-Ab), CD103, SiglecF (BD Bioscience, San Jose, CA), CD11c, IFNy (eBioscience San Diego, CA), CD64 (Biolegend, San Diego, CA). For intracellular cytokine staining, cells were first incubated with PMA $(10 \mathrm{ng} / \mathrm{mL})$ plus ionomycin $(10 \mu \mathrm{M})$, with the addition of Golgi-stop reagent (BD Bioscience, San Jose, CA) for $4 \mathrm{~h}$ at $37^{\circ} \mathrm{C}$. Staining was then performed as above.

T-Distributed Stochastic Nearest-neighbor Embedding (tSNE) was implemented as a plugin in Flowjo $v$ 10.4. Briefly, flow cytometry data for individual samples was concatenated into a single FCS file and a pre-gating strategy was implemented to rid the dataset of cell debris and doublets. Cleaned data were further gated as being Thy $1.2+, \mathrm{IL}-17+$ and the tSNE algorithm was run to separate groups based on the indicated surface markers using the following parameters: perplexity 35 , learning rate 1500 for 3000 iterations.

Microscopic imaging and Histopathologic scoring

Lungs were fixed in $4 \%$ formaldehyde overnight, and $3-\mu \mathrm{m}$ paraffin-embedded sections were stained with hematoxylin and eosin (H\&E). All images were obtained on an Olympus BX-51 microscope. To assess lung injury, slides were scored in a blinded fashion on a 12 point scale which addressed various aspects of histopathology including alveolar congestion, hemorrhage, presence of neutrophils in the airspaces, and degree of interstitial thickening ( $0-3$ points each, based on severity).

For fluorescence imaging of paraffin-embedded lung sections, Sections $(4 \mu \mathrm{m})$ were cut, deparaffinized, and hydrated. Slides were incubated in Target Retrieval Solution (Dako \#S1699) for $30 \mathrm{~m}$ in a pressure cooker, cooled to RT, and blocked in 5\% goat/ $5 \%$ donkey serum in Tris buffered saline with $0.05 \%$ Tween (TTBS). Sections were then incubated with anti-pro-SPC (Millipore \#AB3786) at 1:500, anti-CCSP (Seven Hills \#WRAB-3950) at 1:20,000 and anti-T1a (University of lowa Developmental Studies Hybridoma Bank \#8.1.1) at 1:100 overnight at $4{ }^{\circ} \mathrm{C}$. After washing in TTBS, sections were incubated with anti-rabbit Cy5 (Jackson \#711-605-152) and anti-hamster Cy3 (Jackson \#107-166142) antibodies for $1 \mathrm{~h}$ at RT, followed by DAPI for $10 \mathrm{~m}$. Images were captures on a Nikon A1 inverted confocal microscope at $\times 10$, serial images were tiled together to form a composite image of the entire lung section.

Statistical analyses

Statistical analyses were performed using GraphPad Prism software package (GraphPad Software, La Jolla, CA). Following a normality test, differences between experimental groups were determined using $t$-tests (for comparing two groups) or one-way ANOVA with a Tukey post-test (for comparing 3 or more groups) if normally distributed. Non-normally distributed data were analyzed by Kruskal-Wallis test and Dunn's multiple comparison post test. Differences were considered statistically significant when $p<0.05$. All data are represented as mean \pm SEM. All experiments were repeated, at least, in duplicate with similar results. Pertinent statistical information, i.e. $n$-values and $p$-values, are given in individual figure legends.

\section{RESULTS}

Mice challenged with H1N1, or H1N1/MRSA, exhibit elevated levels of CCL2 in lungs

We utilized a murine model of post-viral bacterial pneumonia. Mice were challenged with 100 pfu of H1N1 i.n. and on day 5 were mock-infected or injected i.t. with $10^{7} \mathrm{cfu}$ of MRSA. Prior infection with influenza has previously been shown, by our group and by others, to result in increased bacterial growth as compared to that seen in non-influenza infected WT mice. ${ }^{16,23}$ All samples in this study were collected on day 6 ( $24 \mathrm{~h}$ after MRSA inoculation) unless otherwise specified. We use the shorthand "H1N1/MRSA" to denote sequential infection of H1N1 then MRSA in this model.

To understand the role of CCR2 in this model we wanted to measure expression of CCL2, which serves as a ligand for CCR2. CCL2 has been shown previously to be overexpressed in mouse lungs infected with influenza pneumonia. ${ }^{8}$ Our data confirm that cells isolated by bronchoalveolar lavage show massive upregulation of CCL2 mRNA following $\mathrm{H} 1 \mathrm{~N} 1$ alone, additionally, mice infected with H1N1/MRSA showed a similar increase in CCL2 mRNA following $24 \mathrm{~h}$ of bacterial challenge (Fig. 1a). Similarly, lung leukocytes show impressive 80 -fold elevations in CCL2 mRNA (Fig. 1b). At the protein level, lung homogenates show a 2-3-fold increase in CCL2 protein in mice infected with H1N1, MRSA, or H1N1/MRSA (Fig. 1c).

Mice deficient in CCR2 have improved survival and bacterial clearance in the lung post-H1N1/MRSA as compared to WT mice We next utilized CCR2 ${ }^{-1-}$ mice to further address the role of CCR2 during H1N1/MRSA dual infection or single infection with either H1N1 or MRSA alone. WT and CCR2 ${ }^{-1-}$ mice were inoculated with $\mathrm{H} 1 \mathrm{~N} 1$ and/or MRSA as described above, and lungs were collected $24 \mathrm{~h}$ post bacterial challenge. Signs of lung injury were apparent following dual infection in WT mice with significant disruption to alveolar epithelial cells as evidenced by fluorescence microscopy for the type 1 alveolar epithelial cell marker T1a, the type II alveolar epithelial cell marker surfactant protein C (pro-spc), and the airway epithelial cell marker CCSP (Fig. 2a, high resolution images are supplied in the supplemental data). Type II epithelial cells were especially affected in dual infected WT mice and displayed a much less intense staining and lower overall numbers than uninfected (Fig. 2a, higher power fields). Patchy type I epithelial cell disruption was also noted (Fig. 2a yellow arrows). CCR2 $2^{-1-}$ mice displayed much less type II disruption than WT mice (Fig. 2a high power fields), however, a similar level of type I cell 

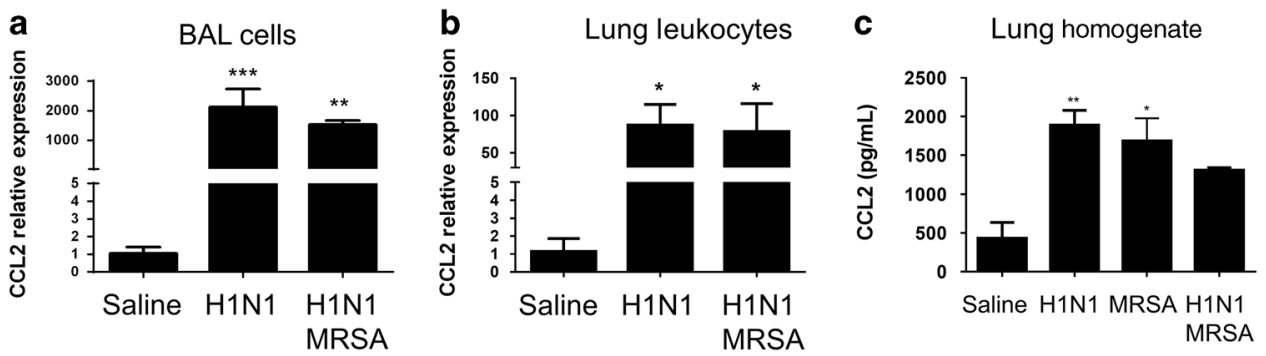

Fig. 1 Expression of CCL2 (MCP-1) in the lung is increased following H1N1 infection or H1N1/MRSA dual infection. a Bronchoalveolar lavage (BAL) was performed on uninfected, H1N1 or H1N1/MRSA dual infected WT mice (6 days after H1N1 infection, $24 \mathrm{~h}$ following MRSA infection). CCL2 expression was measured via qRT-PCR from isolated BAL cells. b Leukocytes were collected from collagenase digested lung tissue $24 \mathrm{~h}$ after MRSA infection (6 days after H1N1 infection). CCL2 expression was measured via qRT-PCR. c Whole-lung homogenates were prepared $24 \mathrm{~h}$ after MRSA infection (6 days following H1N1 infection). CCL2 was quantified by ELISA. Statistical significance was calculated by Kruskal-Wallis non-parametric test, ${ }^{*} p<0.05,{ }^{* *} p<0.01$ versus uninfected. $n=3$ mice per group

disruption was noted (Fig. 2a yellow arrows). When examined for other pathologic features (e.g. hemorrhage and leukocyte infiltration) by $\mathrm{H} \& \mathrm{E}$ staining, lungs from $\mathrm{CCR}^{-/-}$mice exhibited less histopathology overall than WT mice following H1N1/MRSA dual infection (Fig. 2b). Additionally, CCR2 ${ }^{-1-}$ mice demonstrated increased survival in comparison to WT mice following this lethal dual infection (Fig. 2c). To further address the level of lung injury in dual infected mice we scored $\mathrm{H} \& \mathrm{E}$ stained lung sections following H1N1/MRSA dual infection based on various criteria of lung injury including: alveolar congestion, hemorrhage, presence of neutrophils in the airspace, and level of interstitial thickening. Consistent with the increased survival, $\mathrm{CCR}^{-1-}$ mice had lower histopathology scores in comparison to WT mice (Fig. 2d). Additionally, amounts of BALF-associated albumin (a surrogate measurement for lung protein leakage/lung injury) were also decreased in $\mathrm{CCR}^{-1-}$ mice infected with H1N1/MRSA in comparison to WT mice (Fig. 2e). We next measured both viral and bacterial burdens in WT and $\mathrm{CCR}^{-/-}$mice following $\mathrm{H} 1 \mathrm{~N} 1 /$ MRSA dual infection. In comparison to WT mice, levels of MRSA cfu were significantly reduced at $24 \mathrm{~h}$ post-H1N1/MRSA infection and the same trend was evident at $48 \mathrm{~h}$ post infection (Fig. 2f). Similarly, in comparison to WT mice, $\mathrm{CCR}^{-1-}$ mice exhibited much lower viral replication indicated by $M 1$ gene expression at both 24 and 48 hpi ( 6 and 7 days following influenza challenge, respectively) (Fig. 2g). Interestingly, $\mathrm{CCR}^{-1-}$ mice exhibited minimal changes in comparison to WT mice during single infection with H1N1 or alone. Influenza titers were slightly increased in $\mathrm{CCR}^{-1-}$ mice at 5 dpi ( 2-fold Figure S1A) however, this did not reach statistical significance and there was no difference in mortality with both groups reaching euthanasia criteria (percent weight loss) by $8 \mathrm{dpi}$ (Figure S1B). Additionally, as shown in Fig. 3 below, WT and $C C R 2^{-1-}$ mice show similar clearance of MRSA infection alone. Taken together these data surprisingly suggest that $\mathrm{CCR}^{-1-}$ mice handle dual infection better than WT mice.

$\mathrm{CCR}^{-1-}$ mice recruit more neutrophils post-infection

Given that CCR2 generally regulates recruitment of myeloid cells during influenza pneumonia, ${ }^{8,29}$ we next determined the inflammatory cell composition of the BALF or total lung digestions in uninfected mice, mice infected with $\mathrm{H} 1 \mathrm{~N} 1$, or mice dual infected with $\mathrm{H} 1 \mathrm{~N} 1 / \mathrm{MRSA}$. Interestingly, the total number of cells recruited to the lung were not significantly different between the groups in WT vs. CCR2 ${ }^{-1-}$ mice following single or dual infection, although there was a trend for lower numbers in $\mathrm{CCR}^{-1-}$ mice with $\mathrm{H} 1 \mathrm{~N} 1$ alone (Fig. 3a). In response to $\mathrm{H} 1 \mathrm{~N} 1$ alone, monocytes and macrophages predominated in the $\mathrm{B} 6$ mice, whereas $\mathrm{CCR} 2^{-1-}$ mice recruited relatively small numbers of monocytes (Fig. $3 \mathrm{~b}$ ). In contrast, following H1N1/MRSA dual infection both WT and $\mathrm{CCR}^{-1-}$ mice recruited predominantly neutrophils, and, $\mathrm{CCR}^{-1-}$ mice exhibited a trend towards increased neutrophil recruitment in comparison to WT mice (Fig. 3c). Numbers of lymphocytes remained consistent between all groups (Fig. 3d). To assess if neutrophil recruiting chemokines were altered in $\mathrm{CCR}^{-1-}$ mice infected with $\mathrm{H} 1 \mathrm{~N} 1$, we measured expression of both CXCL1 (KC) and CXCL2 (MIP2) in the lungs by qRT-PCR. Following $\mathrm{H} 1 \mathrm{~N} 1$ infection alone, $\mathrm{CCR} 2^{-1-}$ mice displayed a $\sim 3$-fold increase in CXCL1 mRNA expression at both 3 and 5 dpi (Fig. 3e). A similar increase was noted in the expression of CXCL2 mRNA at 3 dpi (Fig. 3f). In agreement with this, amounts of CXCL1 protein, as measured by ELISA, were also elevated in the lungs of H1N1 infected $\mathrm{CCR}^{-1-}$ mice at 3 and 5 dpi (Fig. $3 \mathrm{~g}$ ). Given that the $\mathrm{CCR}^{-1-}$ mice expressed more PMN-recruiting chemokines and had higher PMN accumulation post-dual infection, we wanted to verify that PMNs were playing a key role in host defense against MRSA post-H1N1. Thus, WT and $\mathrm{CCR}^{-1-}$ mice were infected with MRSA alone or H1N1/MRSA in the presence or absence of anti-GR1 neutralization to deplete PMNs. Figure $3 \mathrm{~h}$ demonstrates that antiGR1 treatment to remove PMNs increases the MRSA cfu in the lung.

$\mathrm{CCR}^{-1-}$ mice produce IL-17 in response to H1N1 infection Neutrophils are well documented to be recruited via IL-17. ${ }^{30,31}$ To address whether $\mathrm{CCR}^{-1-}$ mice were producing IL-17 following H1N1 infection, we infected WT or CCR2 $2^{-1-}$ mice i.n. with $100 \mathrm{pfu}$ H1N1. Lungs were collected at $5 \mathrm{dpi}$ and collagenase digested lung tissue was analyzed via flow cytometry in conjunction with t-SNE dimensional reduction which reduces data for multiple fluorophores into a single twodimensional plot. $^{32}$ Four distinct clusters of IL-17 producing lymphocytes were identified, namely, classic Th17 cells (Thy1.2, $\mathrm{CD} 3+, \mathrm{CD} 4+$ ), $\gamma \delta-\mathrm{T}$ cells (Thy1.2+, CD3,$+ \mathrm{CD} 4-, \mathrm{\gamma} \delta-\mathrm{TCR}+$ ), and NKT like lymphocytes (Thy1.2+, CD3+, CD4-, NK1.1+) (Fig. 4a, histograms detailing surface markers of these populations are shown in Figure S2). In comparison to WT H1N1 infected mice, $\mathrm{CCR}^{-/-}$mice recruited $\sim 3$-fold more IL-17 producing $\mathrm{\gamma} \delta$-T cells and $\sim 2.5$-fold more Th17 cells (Fig. 4b). Numbers of IL-17 producing NKT cells were somewhat higher in $\mathrm{CCR}^{-1-}$ mice, however, this change did not reach statistical significance (Fig. 4b). We also detected an interesting population of IL-17, IL-22 co-producing lymphocytes that was increased by $\sim 2$-fold in $\mathrm{CCR}^{-1-}$ mice (Fig. 4b). Interestingly, numbers of recruited IFNY-producing Th1 cells, as well as IFNY-producing CD8+ lymphocytes were not affected with both WT and CCR2 ${ }^{-1-}$ mice recruiting equivalent numbers at 5 dpi (Fig. $4 c-e$ ).

We further measured expression of IL-17 mRNA in lungs of WT and $\mathrm{CCR}^{-1-}$ mice, following $\mathrm{H} 1 \mathrm{~N} 1$ single infection, and postH1N1/MRSA dual infection. CCR2 ${ }^{-1-}$ mice exhibited increased expression of IL-17 at both 3- and 5-days post influenza infection alone (Fig. 4f). Similarly, IL-17 mRNA expression was fourfold higher in $\mathrm{CCR}^{-/-}$lungs after H1N1/MRSA dual infection than in WT lungs (Fig. 4g). These data suggest a role for CCR2+ 
a
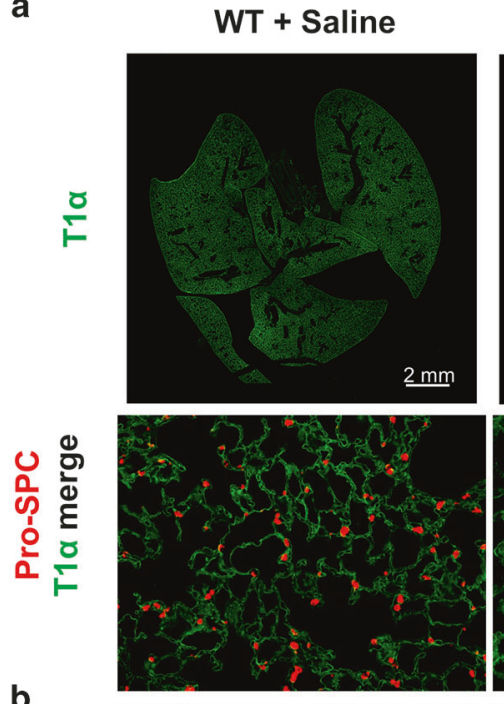

b

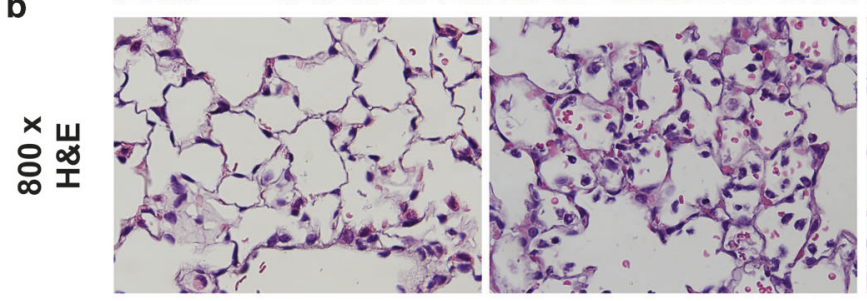

C
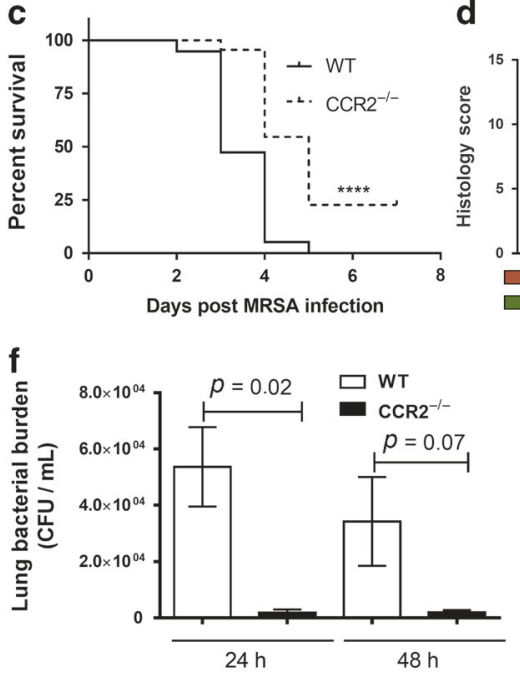

WT + H1N1 / MRSA
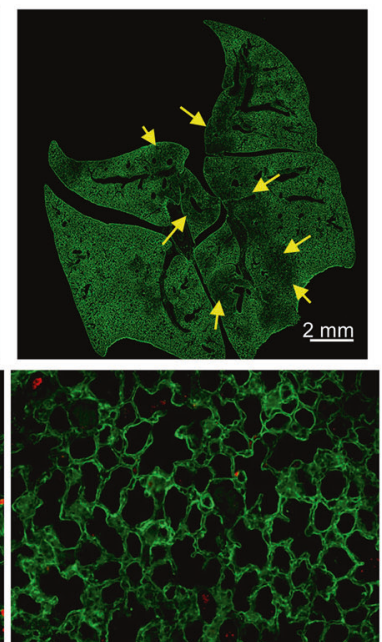
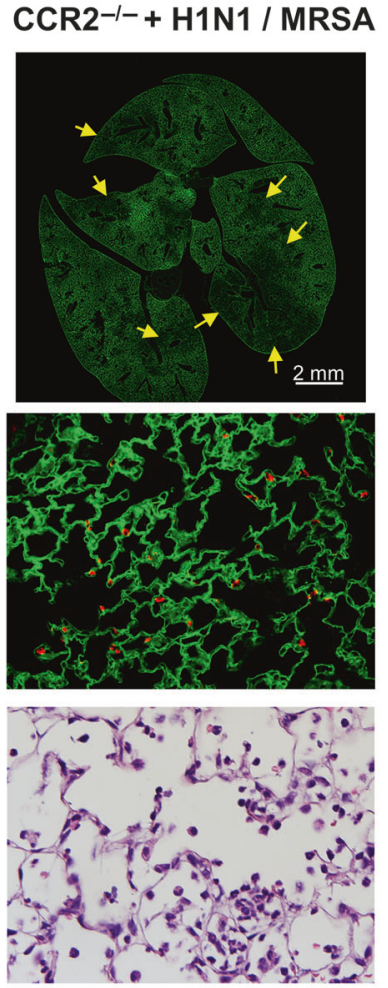

d

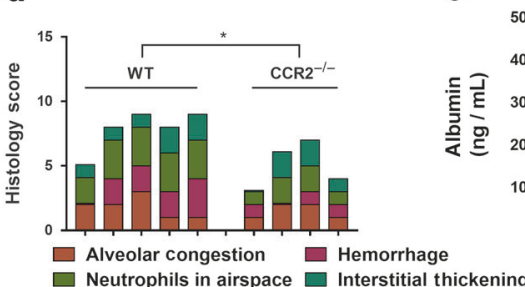

g

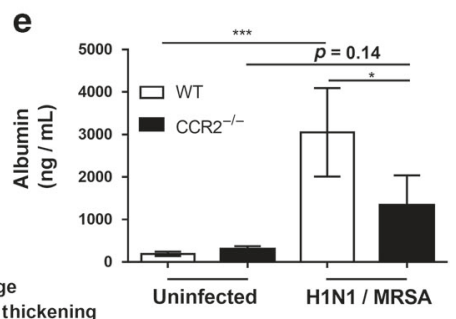

Uninfected H1N1/MRSA

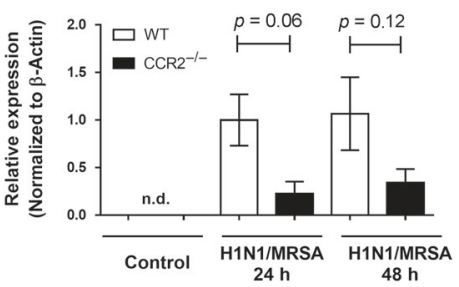

Fig. 2 Loss-of-CCR2 signaling facilitates bacterial clearance and increases survival in H1N1/MRSA dual infected mice. a, b WT or CCR2 ${ }^{-1-}$ mice were infected for $5 \mathrm{~d}$ with $\mathrm{H} 1 \mathrm{~N} 1$ and subsequently infected with MRSA for $24 \mathrm{~h}$. Formalin fixed lung sections were prepared and stained with pro-SPC and T1 $\alpha$ as markers for type 2 and type 1 alveolar epithelial cells, respectively a or H\&E $\mathbf{b}$ (representative images of $n=4$ mice per group). c Survival curves of WT and CCR2 ${ }^{-\prime-}$ mice following H1N1 / MRSA ( $n=19$ mice per group). d Histology scoring of either WT or $\mathrm{CCR}^{-1-}$ mouse infected with H1N1/MRSA ( $n=4-5$ mice per group). e BAL was performed on uninfected or H1N1/MRSA infected mouse groups and albumin was quantified from BALF by ELISA ( $n=3-5$ mice per group). $\mathbf{f}$ Lung CFU from WT mice and CCR2 ${ }^{-1-}$ mice following sequential infection with H1N1 and MRSA. g Expression of the H1N1 M1 gene as measured by qRT-PCR ( $n=3$ mice per group). Statistical significance calculated using ANOVA, ${ }^{*} p<0.05,{ }^{* *} p<0.01,{ }^{* * *} p<0.0001$

monocytes in modulating or regulating the activation of Th17 cells in response to sequential H1N1/MRSA infection.

Lung dendritic cell profiles are different in H1N1 or H1N1/MRSA infected $\mathrm{CCR}^{-1-}$ mice compared to WT mice $\mathrm{CCR}^{+}$monocyte-derived cells have been implicated previously in the immune pathology during influenza infection and CCR2 antagonism offers protection against such pathology. ${ }^{8,33}$ Having seen differences in monocyte numbers in lungs post-H1N1 infection and sequential infection we wanted to characterize the dendritic cell population accumulating in the lung following $\mathrm{H} 1 \mathrm{~N} 1$ infection to assess if there were any differences in numbers of dendritic cells accumulated between infected WT and $\mathrm{CCR}^{-1-}$ mice. WT and CCR2 ${ }^{-1-}$ mice were infected i.n. with 100 pfu H1N1, $5 \mathrm{dpi}$, lungs were collagenase digested and flow cytometry was performed to identify the dendritic cell populations present in the lungs. Conventional DCs were identified as $\mathrm{CD} 11 \mathrm{c}^{+}, \mathrm{MHCll}+$, CD64-, and SiglecF- (Figure S3). To further identify dendritic cell 

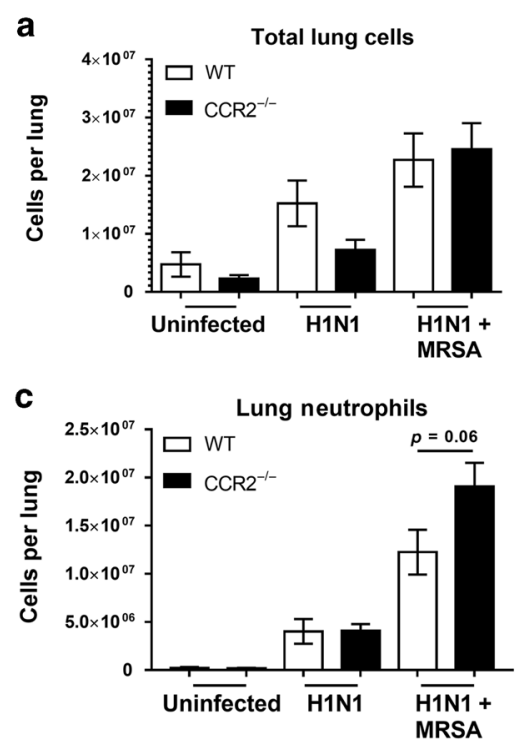

e

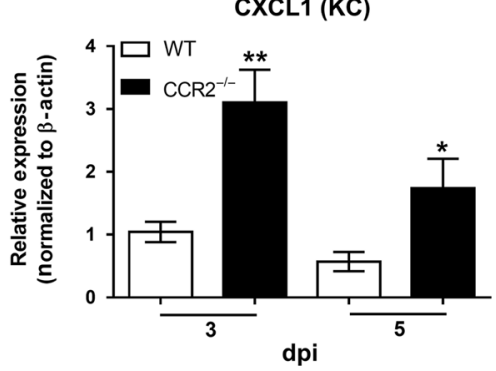

g

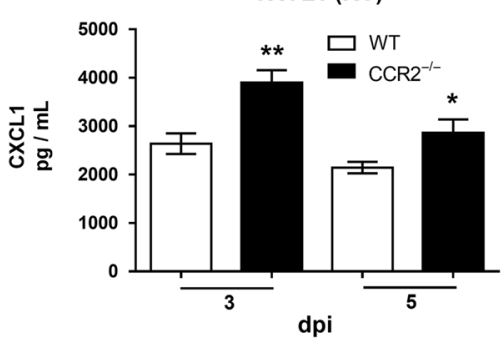

b
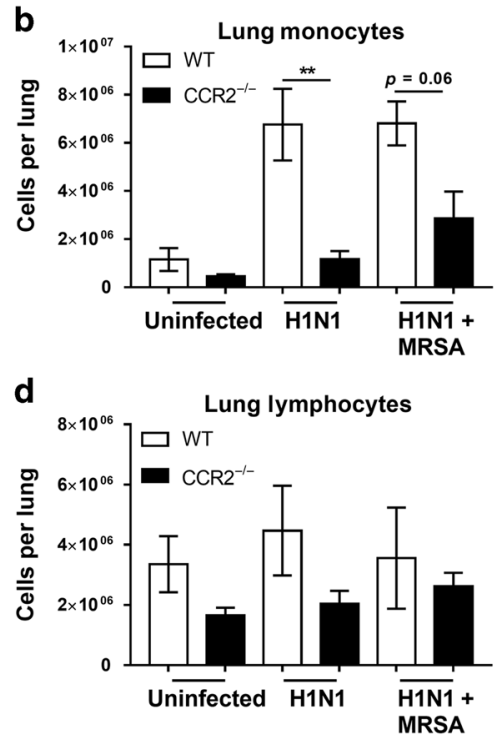

f

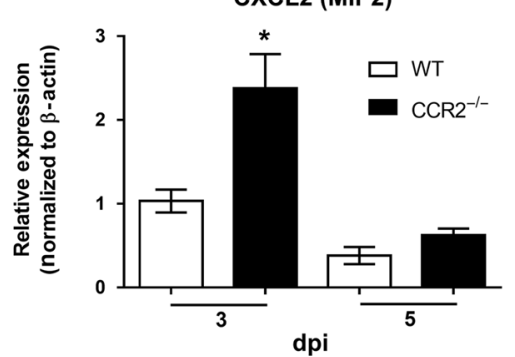

h

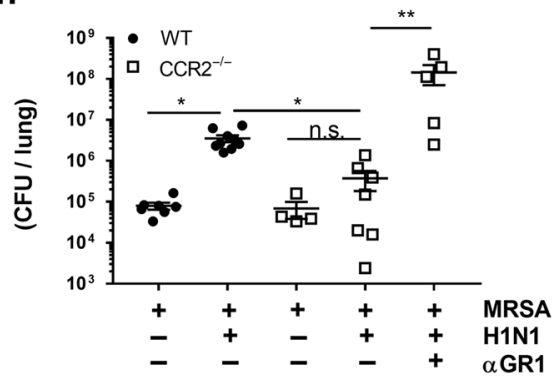

Fig. $3 \mathrm{CCR}^{-1-}$ mice recruit neutrophils instead of monocytes following H1N1/MRSA infection. a Total leukocytes obtained from enzymatically digested lung. b-d Total leukocytes from lung homogenates were prepared for cytospin and counted manually following differential staining. e, $\mathbf{f W T}$ or CCR2 ${ }^{-/-}$mice were infected for 3 or 5 days with H1N1. Total RNA was extracted and analyzed for expression of the indicated transcript. g Lung homogenates were prepared from WT or CCR2 ${ }^{-1-}$ mice infected for 3 or 5 days with H1N1 alone and the amount of CXCL1 protein was determined by ELISA. $\mathbf{h}$ WT or CCR2 ${ }^{-1-}$ mice were infected with the indicated pathogen and treated with $\alpha \mathrm{GR} 1$ antibody to deplete neutrophils, lung bacterial burden was determined by serial dilution of lung homogenate. Statistical significance was determined by ANOVA, or Kruskal-Wallis non-parametric test in $\mathbf{h} .{ }^{*} p<0.05,{ }^{* *} p<0.01$ ( $n=4-9$ mice per group)

subsets, cDCs were gated as being either CD103+ or CD11b+ (Fig. 5a). CCR2 ${ }^{-1-}$ mice exhibited a $\sim 5$-fold increase in the number of CD103+ DCs present in the lungs at 5 dpi (Fig. 5b). Additionally, $\mathrm{CCR}^{-1-}$ mice displayed a $\sim 2$-fold reduction in the number of CD11b DCs (Fig. 5c). We next isolated total CD11c+ cells from the lungs of WT or $C C R 2^{-/-}$mice that were uninfected or following $\mathrm{H} 1 \mathrm{~N} 1$, or H1N1/MRSA, infection and analyzed them for expression of pro-Th17 transcripts. CD11c+ cells isolated from $\mathrm{CCR}^{-1-}$ mice exhibited significant increases in both IL- 6 and IL-23 following H1N1 infection in comparison to WT CD11c+ cells (Fig. 5d, e). No change in IL-1 $\beta$ transcript was detected in $\mathrm{CCR}^{-/-}$mice in comparison to WT; however, both groups showed elevated IL-1 $\beta$ in comparison to uninfected control groups (Fig. $5 f$ ). Similar trends in IL-6 and IL-23 expression were also noted following H1N1/MRSA dual infection (Fig. 5g, h). Additionally, CD11c+ cells isolated from dual infected $\mathrm{CCR}^{-1-}$ mice displayed a $\sim 2$-fold reduction in IL-27 transcript expression (Fig. 5i). These data strongly suggest that resident $\mathrm{CD} 103+\mathrm{DCs}$ in $\mathrm{CCR}^{-/-}$mice are responsible for skewing effector T-cell responses toward Th17. Additionally, the cytokine milieu is likely to influence IL-17 production by other lymphocyte subsets as well.

Influenza induces expression of IL-17 receptor on lung epithelial cells to promote CXCL1 induction

Given that $\mathrm{CCR}^{-1-}$ mice express more IL-17, we tested the influence of influenza on expression of IL-17R on lung epithelial cells. Type II epithelial cells were isolated from both WT and $\mathrm{CCR} 2^{-1-}$ mice and infected in vitro with $\mathrm{H} 1 \mathrm{~N} 1$, at a $\mathrm{MOI}$ of 0.01 for $48 \mathrm{~h}$ and expression of IL-17 receptor was subsequently analyzed by qRT-PCR. Interestingly, H1N1 infection upregulated expression 
a

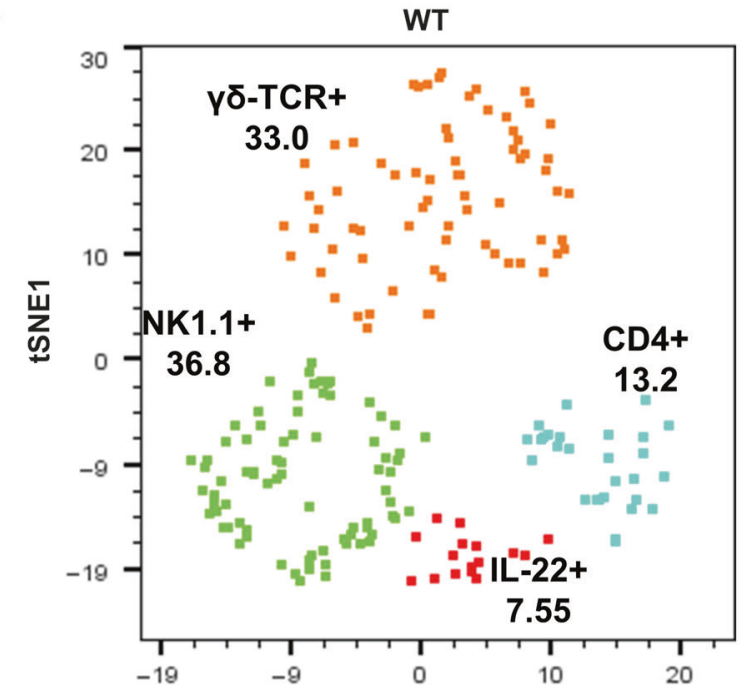

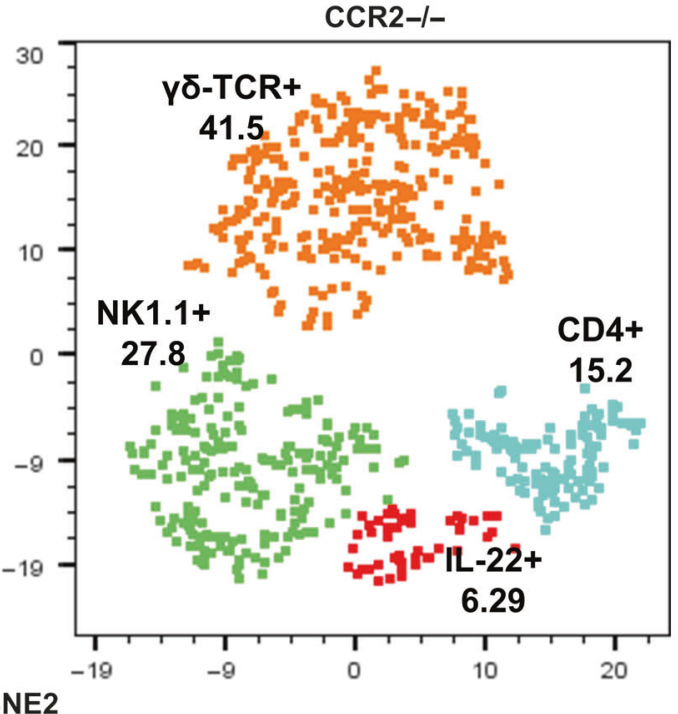

b $\gamma \delta \mathrm{TCR}+$ cells
$($ Thy1.2+, $\gamma \delta \mathrm{TCR}+, \mathrm{CD} 3+, \mathrm{CD} 4-\mathrm{IL}-17+)$ Th17 cells CD4+, CD3+, IL-17+) (Thy1.2+, NK1.1+ cells

$\mathrm{IL}-22+, \mathrm{IL}-17+$ cells
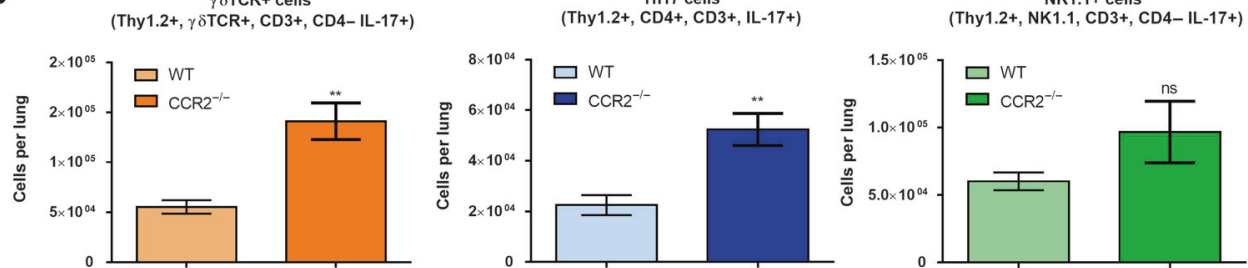

(Thy1.2+, $\gamma \delta \mathrm{TCR}+, \mathrm{CD} 3+$, IL-22+, IL-17+)
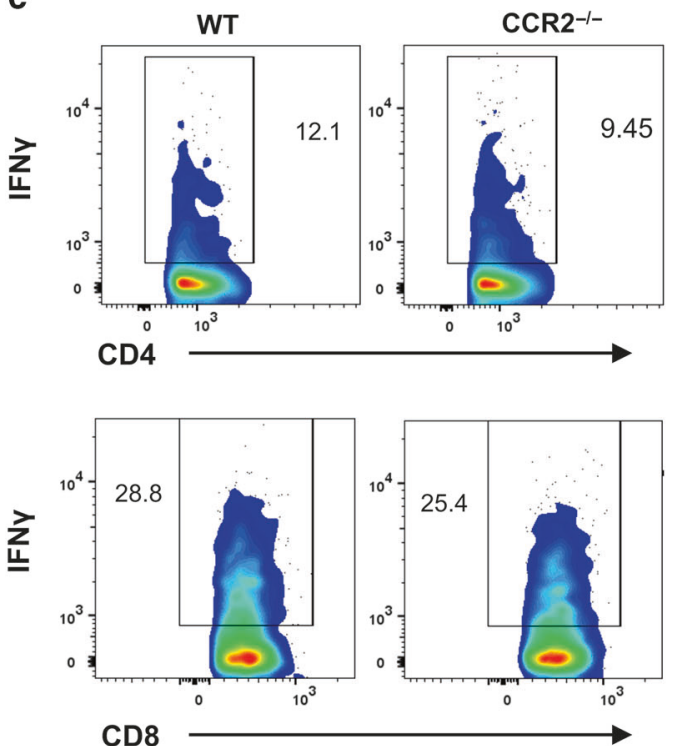

d

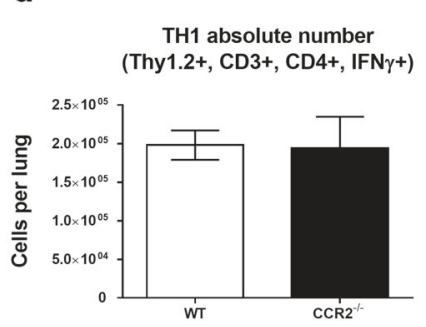

e

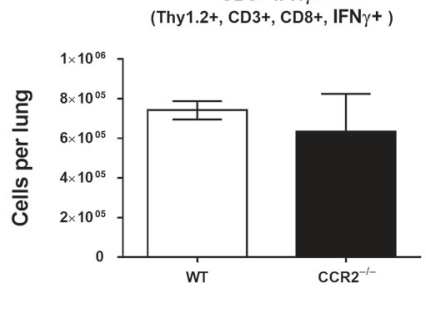

f

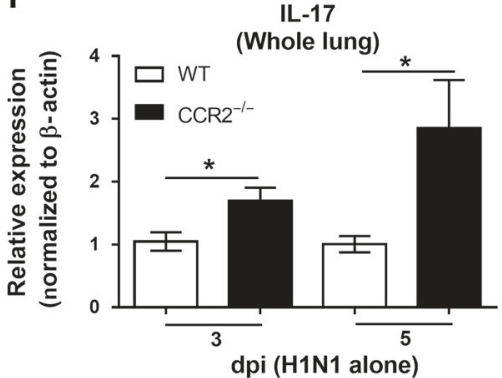

g

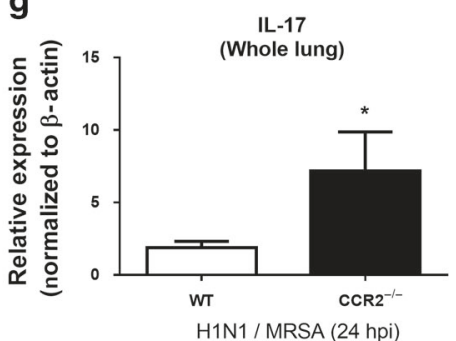

Fig. 4 Abrogation of CCR2 signaling results in increased infiltration of IL-17 producing lymphocytes and increased expression of IL-17 in the lungs following H1N1 or H1N1/MRSA dual infection. a t-SNE multidimensional reduction of IL-17+ lymphocytes isolated from the lungs of WT or CCR2 ${ }^{-1-}$ mice at $5 \mathrm{dpi}(\mathrm{H} 1 \mathrm{~N} 1$ single infection). b Quantification of the four distinct IL-17 producing populations identified in a. c Representative flow plots showing percentage of either CD4+, IFN $\gamma+$ (top), or CD8+, IFN $\gamma+$ (bottom) lymphocytes in the lungs of mice infected with $\mathrm{H} 1 \mathrm{~N} 1$ for $5 \mathrm{~d}$. d, e Quantification of lymphocytes from c. $\mathbf{f}$ WT or CCR2 ${ }^{-1-}$ mice were infected with $\mathrm{H} 1 \mathrm{~N} 1$, at 3 or $5 \mathrm{dpi}$ whole-lung homogenates were prepared and expression of IL-17 mRNA was determined via qRT-PCR. g WT or CCR2 ${ }^{-/-}$mice were infected with H1N1 for $5 \mathrm{~d}$ and then subsequently infected with MRSA, $24 \mathrm{~h}$ post MRSA infection, lung homogenates were prepared and expression of IL-17 transcript was determined via qRT-PCR. Statistical significance was calculated by Student's $t$-test $\mathbf{b}, \mathbf{d}, \mathbf{e}$ and $\mathbf{g}$ or ANOVA $\mathbf{f}^{*} p<0.05,{ }^{* *} p<0.01$, $n=3-5$ mice per group

of IL-17R in epithelial cells from both genotypes, but there was no differential expression between WT and CCR2 ${ }^{-1-}$ mice (Fig. 6a). When expression of CXCL1 was assessed in epithelial cell cultures infected with H1N1 in the presence and absence of IL-17, we found that the addition of either IL-17 or $\mathrm{H} 1 \mathrm{~N} 1$ alone was sufficient to increase CXCL1 expression by AECs, however, an additive increase in CXCL1 expression was observed when AECs were administered both IL-17 and H1N1 simultaneously (Fig. 6b). 
a

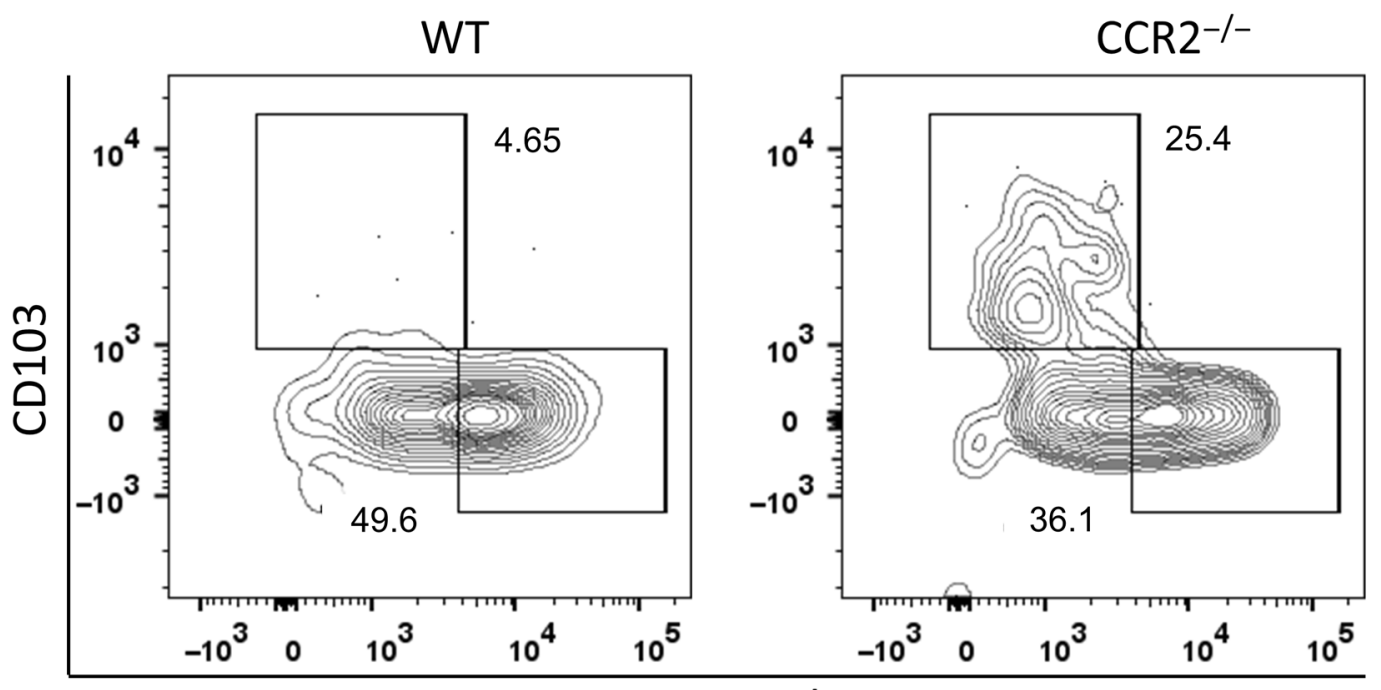

$\mathrm{CD} 11 \mathrm{~b}$

b

CD103+ DCs

(CD45+, MHCII+, CD11C+, CD11b-, CD103+)

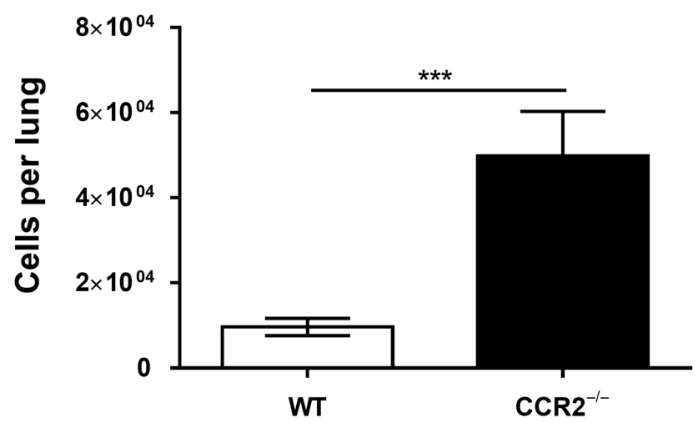

d

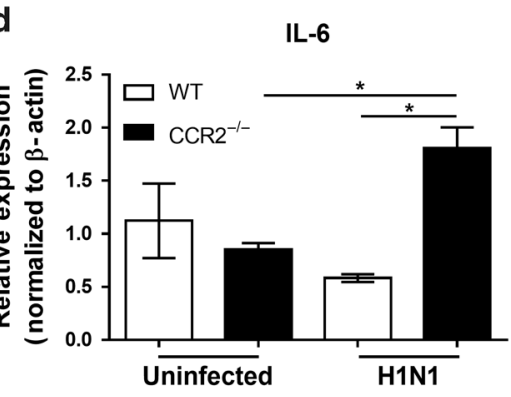

e

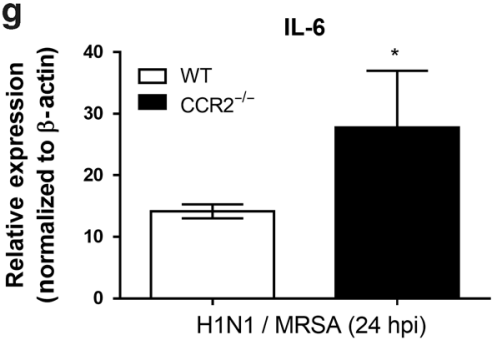

C

CD11b+ DCs

(CD45+, MHCII+, CD11C+, CD11b+, CD103-)

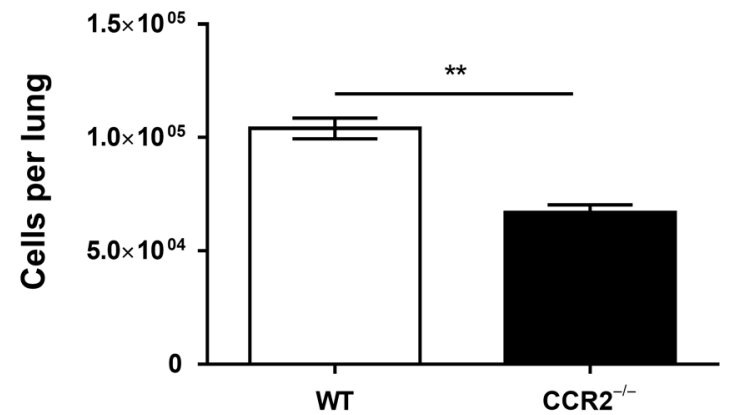

IL-23

f

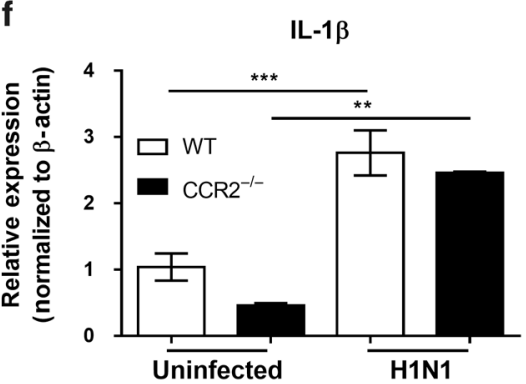

i

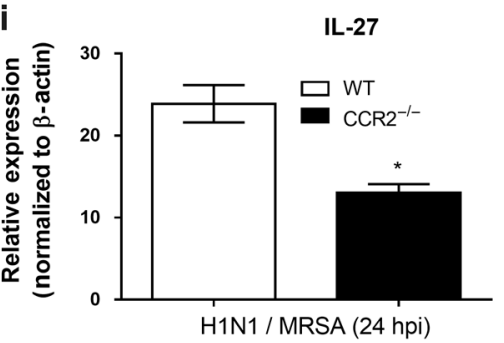

Fig. $5 \mathrm{CCR}^{-/-}$mice accumulate more CD103+, pro-IL-17, dendritic cells to the lungs. a WT or CCR2 ${ }^{-1-}$ mice $(n=5$ per group) were infected with H1N1. 5 dpi, lungs were collected and leukocyte populations were analyzed by flow cytometry for the presence of either CD103+ or CD11b + dendritic cells, shown are representative flow plots from two separate experiments. $\mathbf{b}$, $\mathbf{c}$ Quantitation of flow cytometry data in a. d-f WT or CCR2 ${ }^{-1-}$ mice ( $n=3$ per group) were infected with H1N1, 5 dpi lungs were collected and collagenase digested. Total CD11c + cells were isolated via magnetic sorting and analyzed by qRT-PCR for the indicated transcript. $\mathbf{g}-\mathbf{i}$ Mice ( $n=5-7$ per group) were infected with H1N1 for 5 days and then subsequently infected with MRSA for $24 \mathrm{~h}$. Total CD11c + cells were isolated from collagenase digested lungs via magnetic sorting and analyzed for the indicated transcript by qRT-PCR. Statistical significance was calculated using ANOVA d, e and f or Student's $t$-test $\mathbf{g}, \mathbf{h}$, and $\mathbf{i},{ }^{*} p<0.05,{ }^{* *} p<0.01,{ }^{* * *} p<0.001$ 
a

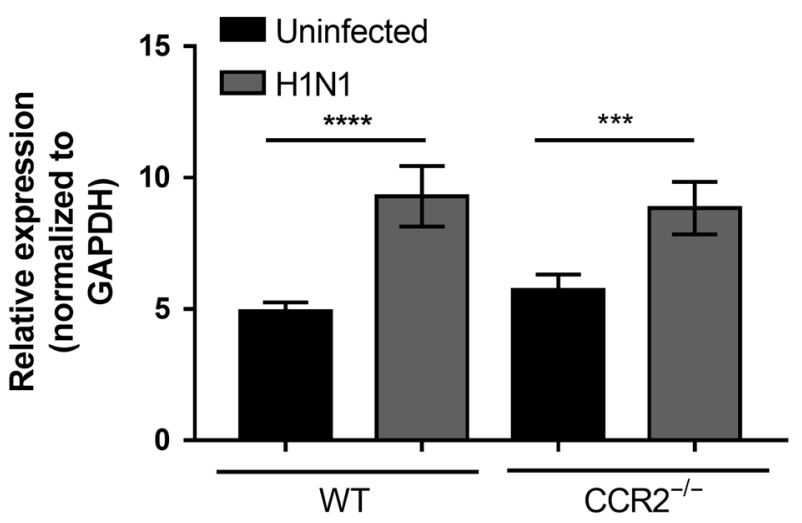

b

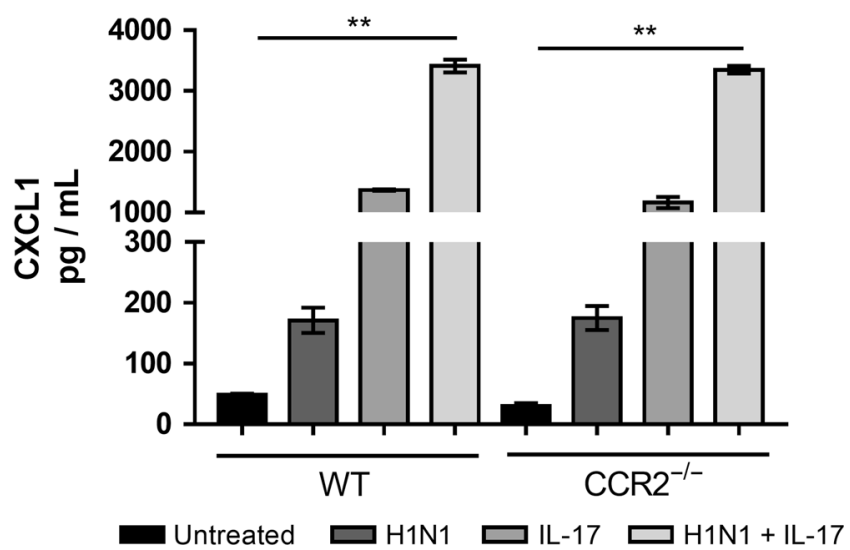

Fig. 6 Isolated alveolar epithelial cells express IL-17 receptor and secrete CXCL1 in response to both IL-17 stimulation and H1N1 infection. a Type II alveolar epithelial cells were isolated from either WT or CCR2 ${ }^{-}$mice and infected with H1N1 $(\mathrm{MOI}=0.01)$ or left uninfected for $48 \mathrm{~h}$. Total RNA was extracted and analyzed for the expression of IL-17R $\alpha$. $\mathbf{b}$ Type II alveolar epithelial cells were treated either with recombinant IL-17 $(10 \mathrm{ng} / \mathrm{mL}), \mathrm{H} 1 \mathrm{~N} 1(\mathrm{MOI}=0.01)$, or a combination of $\mathrm{IL}-17$ and $\mathrm{H} 1 \mathrm{~N} 1$. After $48 \mathrm{~h}$ of treatment supernatants were analyzed for CXCL1 by ELISA. Statistical significance was determined using ANOVA a, or Kruskal-Wallis non-parametric test $\mathbf{b}{ }^{*} p<0.05,{ }^{* *} p<0.01,{ }^{* * *} p<0.001,{ }^{* * *} p<0.0001, n=3-5$ biological replicates per group

Antagonism of CCR2 in WT mice improves bacterial clearance and elicits a pro-Th17 response

To determine whether antagonism of CCR2 held therapeutic potential in our model of post-viral bacterial pneumonia, we treated WT mice with a small molecule inhibitor of CCR2, PF04178903 , twice daily for 5 days from day 0 through day 5 postH1N1 and prior to MRSA infection. On day 6, lung homogenate cultures isolated from WT mice treated with PF-04178903 compared to untreated WT mice demonstrated tenfold lower bacterial cfu of MRSA, respectively (Fig. 7a). Consistent with the $\mathrm{CCR}^{-1-}$ mice, PF-04178903 treated mice had a marked increase in segmented neutrophil infiltration to the lung following MRSA infection (Fig. 7b). In conjunction with lower bacterial counts in lungs, treated WT mice had increased expression of the pro-Th17 genes IL-1 $\beta$ and IL-6 (Fig. 7c). Additionally, IL-17a, and the neutrophil attracting chemokines $\mathrm{CXCL1}$ and $\mathrm{CXCL2}$ were also increased in CCR2 antagonist treated mice (Fig. 7c).

To further implicate IL-17 as a mechanism of protection in $\mathrm{CCR}^{-1-}$ mice, we treated CCR2 ${ }^{-1-}$ mice with a neutralizing IL-17 antibody in comparison to isotype antibody. Anti-IL-17 antibody was purified from rabbit serum and injected intraperitoneally $6 \mathrm{~h}$ prior to MRSA infection in our model. Unfortunately, we only obtained enough antisera to treat 2 mice per group with $200 \mu \mathrm{g}$ anti-IL-17 or control antibody (Figure S4); however, there was a trend towards IL-17 neutralization impairing bacterial clearance in $\mathrm{CCR}^{-/-}$mice $(p=0.01)$.

\section{DISCUSSION}

The results of earlier studies have shown specific influenza A virus subtypes modulate innate immune responses, including antigen presenting cell populations, pattern-recognition receptor activation, recruitment of cells via chemotaxis, and cytokine expression. ${ }^{34-37}$ These mechanisms not only worsen morbidity and mortality from immunopathology, but also increase susceptibility to secondary bacterial infection after influenza pneumonia. In our study, expression of CCL2 was dramatically increased following H1N1/MRSA (Fig. 1) in wild-type mice and this was associated with recruitment of monocytes and neutrophils (Fig. 3). Interestingly however, $\mathrm{CCR}^{-1-}$ mice were better protected from secondary bacterial pneumonia following influenza and displayed much lower levels of epithelial disruption and lung pathology (Fig. 2a, d) as well as increased survival and greatly augmented bacterial clearance as compared to WT mice (Fig. 2c, f). Innate immune cells are responsible for clearance of MRSA in the first $24 \mathrm{~h}$ post infection; yet, wild-type mice ineffectively clear bacteria in the first $24 \mathrm{~h}$ of infection post-H1N1. Further, this defect correlates with increased levels of CCL2 suggesting that recruitment of CCR2 ${ }^{+}$ monocyte-derived DCs and exudative macrophages not only contribute to immunopathology and mortality but are surprisingly associated with higher bacterial burden in lung.

$\mathrm{CCR}^{+}$monocytes have previously been implicated in the pathogenesis of pneumonia associated with single influenza infection and blockade of CCR2 signaling using a CCR2 antagonist significantly increased survival against influenza singlechallenge. ${ }^{33}$ More recently, influenza $A$ was found to drive robust CCL2 (MCP-1) expression in juvenile mice and this expression was correlated with increased lethality of influenza in comparison to aged mice. ${ }^{9}$ Our results suggest that CCR2 signaling is similarly pathogenic in the setting of H1N1/MRSA dual infection.

Here we present data that loss-of-CCR2 results in increased neutrophil accumulation in response to MRSA following H1N1 infection (Fig. 3c). Paradoxically, CCR2 has been shown to be expressed by, at least, some subsets of neutrophils. ${ }^{38,39}$ However, the neutrophil attracting chemokines CXCL1, and CXCL2 (KC and MIP-2) were similarly upregulated following H1N1/MRSA infection in $\mathrm{CCR}^{-1-}$ mice (Fig. 3e-g) and thus neutrophil recruitment was probably mediated through the more canonical CXCL1/2-CXCR2 axis. Interestingly neutrophils, not inflammatory monocytes, have been shown to be important for the clearance of $S$. aureus; however, $\mathrm{CCR}^{+}$monocytes are important for wound resolution following bacterial clearance. ${ }^{40}$ Furthermore, clearance of $S$. pneumoniae, another common cause of post-influenza secondary bacterial pneumonia, was also shown to rely in part on neutrophil mediated mechanisms. ${ }^{41}$ In support of this, we depleted neutrophils in $\mathrm{CCR} 2^{-1-}$ mice by i.p. injection of aGR1 neutralizing antibody and observed a 100-fold increase in bacterial burden in treated mice indicating that neutrophils are the major clearance mechanism of MRSA following H1N1 infection (Fig. 3h). Thus, it is possible that one reason the CCR2 $2^{-1-}$ mice do better is because their lungs essentially only have neutrophils present to fight the bacterial infection; whereas, the wild-type lungs would have a mixture of both monocytes and neutrophils and the increased cfu in these mice may reflect the poorer functionality of the monocytes relative to the neutrophils. We compared lung derived (following LPS stimulation) neutrophils isolated from CCR2 ${ }^{-1-}$ mice to WT neutrophils in both their ability to phagocytize and to kill MRSA bacterium ex vivo. No difference in phagocytic capacity was noted between $\mathrm{CCR}^{-1-}$ and WT neutrophils; however, there 
a

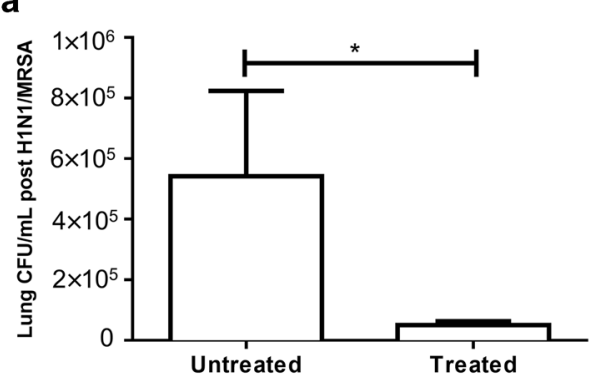

C

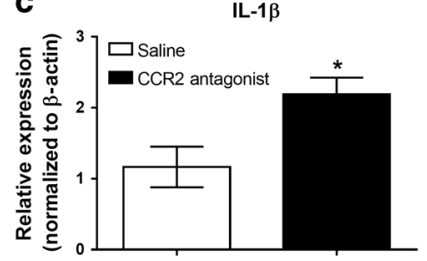

CXCL1 (KC)

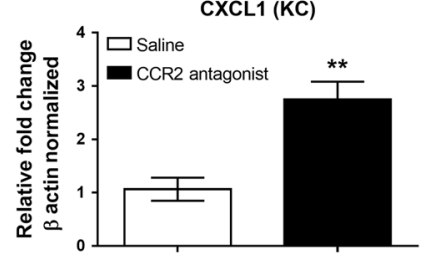

b

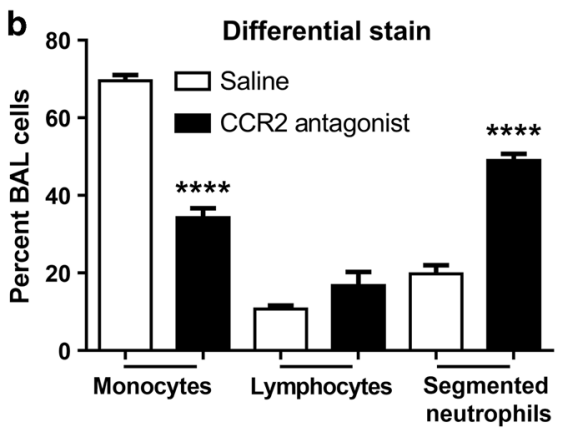

IL6
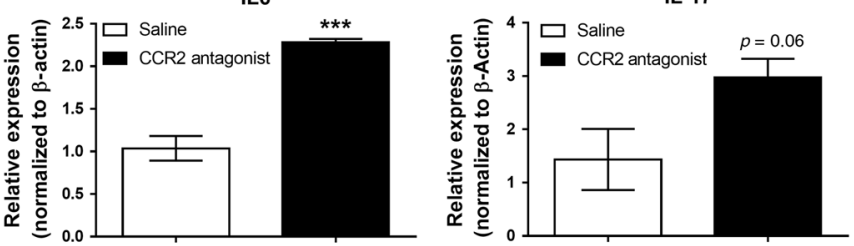

CXCL2 (MIP-2)

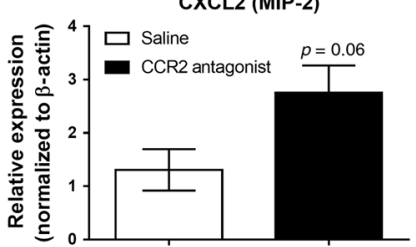

Fig. 7 Mice treated with a CCR2 antagonist have altered cytokine profiles and increased IL-17 expression following H1N1/MRSA dual infection. WT mice were treated with CCR2 antagonist $(50 \mu \mathrm{g}$, subcutaneously) twice daily for 5 days during infection with H1N1. After 5 days of H1N1 infection mice were subsequently infected with MRSA for $24 \mathrm{~h}$. a Lung bacterial burden was calculated by serial dilution of whole lung homogenates $24 \mathrm{~h}$ after MRSA infection. b Following MRSA infection, BAL was performed and numbers of leukocytes were quantified by cytospin of BALF and differential stain. c Lungs were collected and the expression of the indicated transcript was detected by qRT-PCR. Statistical significance was calculated with ANOVA b or Student's $t$-test $\mathbf{a}, \mathbf{c},{ }^{*} p<0.05,{ }^{* *} p<0.01,{ }^{* * *} p<0.001,{ }^{* * * * *} p<0.0001 n=4$ mice per group

was a small but statistically significant increase in the ability of $\mathrm{CCR}^{-1-}$ neutrophils to kill MRSA (Figure S5). It should be noted that $\mathrm{CCR}^{+}$monocytes are important for the clearance of, at least, some bacterial infections. Specifically, depletion of $\mathrm{CCR}^{+}$cells resulted in increased growth and increased lethality, to certain strains, in a mouse model of pulmonary Klebsiella pneumoniae infection. $^{11,13}$ Furthermore, at least one study found that lung specific overexpression of CCL2 enhanced immune responses towards S. pneumoniae; however, CCR2 neutralization had no effect on bacterial killing in an H1N1/S. pneumoniae model of secondary bacterial pneumonia. ${ }^{14,33}$ Thus, while our data indicate that loss-of-CCR2 signaling enhances neutrophil recruitment, neutrophil associated bacterial killing, and confers protection from secondary MRSA-associated pneumonia, further studies must necessarily examine these effects with regards to other bacterial infections.

In the influenza immunity field, there is ample evidence implicating the viral infection in altering the cytokine profile "bridge" that links the innate and adaptive immune responses. Several reports have shown increased expression of IL-12, IFN- $\gamma$, $\mathrm{IL}-2$, and TNF- $a$, which are all markers of Th1 polarization that are present after influenza pneumonia. ${ }^{12,36,42-44}$ Morbidity and mortality have been primarily attributed to immunopathology in the context of a Th1 response. ${ }^{8}$ In bacterial superinfection with S. aureus, the type 1 response and associated immunopathology is amplified. However, beneficial Th17 immunity is suppressed in these mouse models. Robinson et al. rescued protective Th17 pathway activation, increased survival, decreased bacterial burden, and reduced lung injury with administration of exogenous IL-1 $\beta$, an activator of Th17 differentiation and thus production of Th17 associated cytokines like IL-17 and IL-22. ${ }^{22}$ Separately, Kudva et al. demonstrated downstream effectors of the Th17 pathway such as antimicrobial peptides (AMPs) are essential to defense against $S$. aureus pneumonia after influenza challenge. ${ }^{16}$ In both studies, other markers of Th17 including IL17 and IL-23 were present. We previously published that loss-ofCCR2 signaling increased Th17 differentiation and IL-17 production in response to $\gamma$-herpesviruses. ${ }^{26}$ In agreement with this, $\mathrm{CCR}^{-/-}$mice recruited increased numbers of $\mathrm{IL}-17$ secreting lymphocytes in response to $\mathrm{H} 1 \mathrm{~N} 1$ infection (Fig. 4a, b) and had increased expression of IL-17 in the lungs following either H1N1 or H1N1/MRSA infection (Fig. 4f, g). Interestingly, multiple subsets of IL-17 secreting cells were increased in CCR2 ${ }^{-/-}$mice, including, classic Th17 cells, and the more innate-like $\gamma \delta-T$ cells and NK1.1+ NKT cells (Fig. 4a). Both $\gamma \delta-T$ cells and NKT cells are rapidly recruited to the lung following $S$. aureus infection and play a role in host defense through secretion of IL-17. ${ }^{45,46} \mathrm{We}$ also detected a subpopulation of IL-17/IL-22 co-producing cells that was markedly upregulated in $\mathrm{CCR}^{-1-}$ mice following H1N1/MRSA infection. IL-22 has been implicated as a negative regulator of $S$. aureus colonization. ${ }^{47}$ Further, induction of IL-17 and IL-22 by IL-1 $\beta$ was previously shown to be protective in an H1N1/MRSA model via upregulation of the anti-microbial peptide Reglll $\beta^{22}$ Thus, it is likely that the cytokine profile of the DCs are impacting development of both classic Th17 cells as well as IL-17-expressing innate lymphocytes. Importantly, numbers of interferon- $\gamma$ secreting Th1 and CD8+ cells were not altered in $C C R 2^{-1-}$ mice indicating that anti-viral responses were intact (Fig. 4c, d). Interestingly, there is some evidence that both IL-17 and IFN $\gamma$ are necessary for effective clearance of $S$. aureus and $\mathrm{IFNyRI}{ }^{-1-} / \mathrm{IL}-17 \mathrm{Ra}^{-1-}$ dual knockout mice have defects in neutrophil function that render them more susceptible to $S$. aureus infection. ${ }^{48}$ Thus, genetic ablation of CCR2 appears to augment recruitment of IL-17 secreting lymphocytes, 


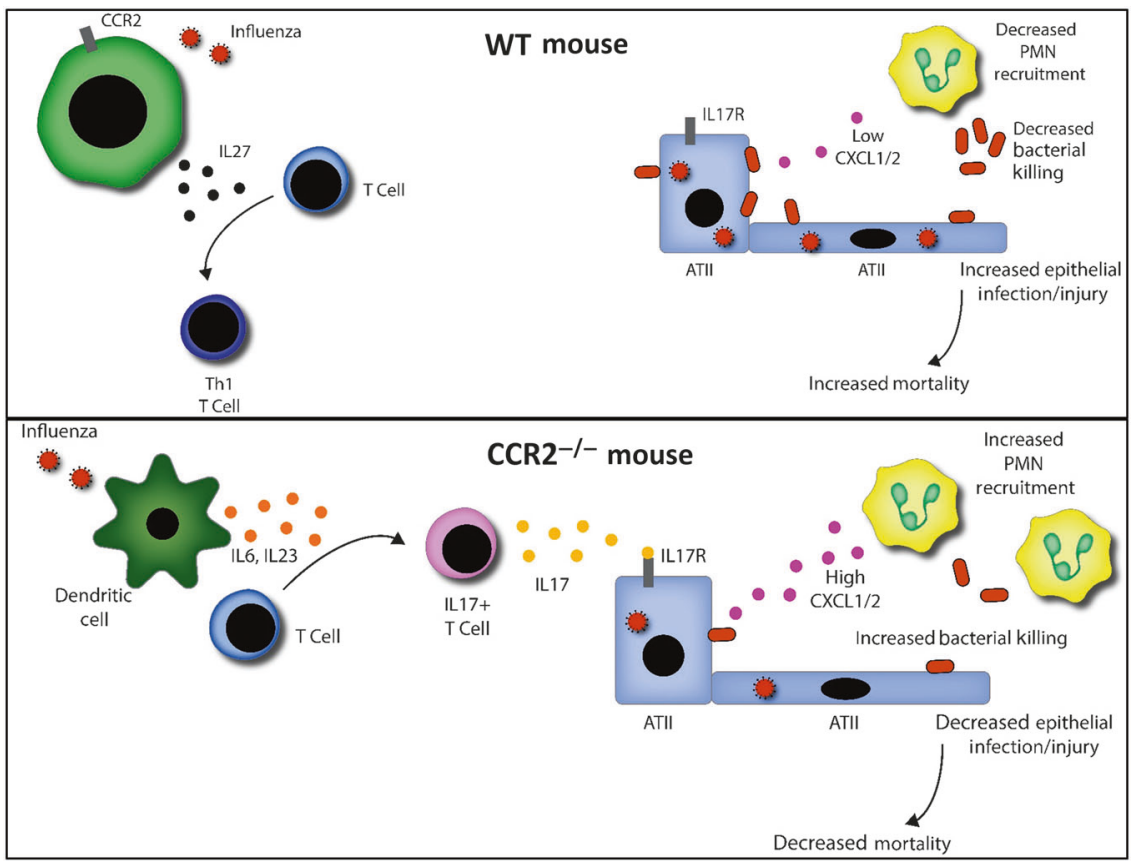

Fig. 8 Proposed model for how CCR2 deficiency mediates increased neutrophil accumulation and bacterial killing. In the absence of CCR2+ monocytes, CD103+ dendritic cells respond to influenza by producing high levels of IL-6, IL-23, and IL-1 $\beta$. These pro IL-17 cytokines in conjunction with decreased production of IL-27 result in robust skewing of Th17 cells as well as IL-17 secreting $\gamma \delta$ T-cells and iNKT cells. IL-17 can activate type II alveolar epithelial cells to produce the neutrophil recruiting chemokines CXCL1/2 which promote the infiltration of bactericidal neutrophils into the lung in response to a subsequent MRSA infection

whereas maintaining production of IFN increased capacity to clear S. aureus bacteria.

To identify the key leukocytes responsible for the protective $\mathrm{CCR}^{-1-}$ phenotype, we performed flow cytometry on cells isolated from Percoll gradients of enzymatically digested lung. In the context of $\mathrm{H} 1 \mathrm{~N} 1$ infection, $\mathrm{CCR} 2^{-1-}$ mice accumulated an increased number of CD103+ DCs (Fig. 5b) as well as reduced numbers of $C D 11 b+$ DCs than WT mice (Fig. $5 c$ ) in the lung. Intestinal CD103+ DCs have previously been shown to be major producers of IL-23 in response to bacteria. ${ }^{49}$ Moreover lung CD103+ DCs have been shown to play a critical role in induction of influenza virus-specific $\mathrm{CD}^{+}{ }^{+}$cells. $^{50}$ In support of our observation that altered DC subsets which accumulate in the lungs $\mathrm{CCR}^{-/-}$mice can drive Th17 differentiation and IL-17 production, we report that $\mathrm{CD} 11 \mathrm{c}+$ cells isolated from the lungs of $\mathrm{CCR}^{-1-}$ mice infected with either H1N1 alone, or H1N1/MRSA exhibited a pro-Th17 cytokine profile with increased expression of IL-6 and IL-23 in comparison to infected WT mice (Fig. 5d, e, g, and h) as well as elevated levels of IL-1 $\beta$ in comparison to uninfected mice (Fig. 5f). We also report that IL-27 is expressed at a lower level in the lungs of CCR2 ${ }^{-1-}$ mice compared to WT mice (Fig. 5i). Expression of IL-27 has previously been linked to increased susceptibility to secondary bacterial pneumonia following H1N1 infection. ${ }^{51}$ IL-27 has also been implicated as a suppressor of Th17 differentiation and there is evidence to suggest that CD11b+ DCs are responsible for IL-27 secretion. ${ }^{52,53}$ Thus, we hypothesize that the alterations in the ratios of CD103+ to CD11b+ DC subsets are responsible for increased expression of IL- 6 and IL-23 along with abrogated expression of IL-27 in the absence of CCR2. CD103+ DCs are likely unaffected by loss of CCR2 as they are the resident DC population in the lung whereas CD11b+ DCs utilize CCR2 for migration to the lung. ${ }^{54,55}$ Interestingly, infection with $\mathrm{H} 1 \mathrm{~N} 1$ alone increased expression of the IL-17 receptor on AECs and coincubation of AECs with both H1N1 and IL-17 greatly increased CXCL1 expression (Fig. 6a, b). However, the DC subsets that are recruited in response to $\mathrm{H} 1 \mathrm{~N} 1$ in WT mice are insufficient to drive robust Th17 differentiation and may in fact actively suppress it, acting as a detriment to bacterial clearance.

To implicate IL-17 as the mechanism for improved bacterial clearance in $\mathrm{CCR}^{-1-}$ lungs compared to WT, we performed IL-17 neutralization studies. Inhibition of IL-17 in CCR2 ${ }^{-1-}$ mice using $i$. p. administration of purified rabbit-anti-mouse IL-17a antibody prior to MRSA infection increased bacterial burden in $\mathrm{CCR} 2^{-1}$ lungs relative to lungs of $\mathrm{CCR}^{-1-}$ mice treated with isotype control antibody (Figure S4). We further validated this finding by inhibiting CCR2 in WT mice using a small molecule compound, PF04178903, injected twice daily for 5 days starting on day of H1N1 inoculation. This compound has been used previously by Lin et al. to antagonize CCR2 in a murine model of influenza pneumonia. ${ }^{33}$ In their study, CCR2 inhibition reduced immunopathology in an influenza pneumonia model. We show that antagonism of CCR2 in a post-influenza pneumonia model improves MRSA clearance associated with increased expression of IL-17 (Fig. 7).

Our conclusion is that CCR2-dependent recruitment of DCs/ macrophages during post-influenza MRSA pneumonia contributes to inhibition of Th17 and innate IL-17-expressing cells, and this process has profound negative effects on bacterial clearance in the lung (summarized in Fig. 8). The data presented in this manuscript provide further evidence that the Th17 pathway is crucial in opposing the immunomodulatory effects of influenza that confer susceptibility to bacterial superinfection, lung injury, and death. We believe that antagonizing CCR2 would provide a potential therapeutic target and significantly decrease mortality and morbidity associated with influenza and post influenza bacterial pneumonia.

\section{ACKNOWLEDGEMENTS}

This work was supported by NIH grants HL127805, HL115618, and Al117229 to B.B.M. Grant support: HL119682, Al117229 (B.B.M.). 


\section{AUTHOR CONTRIBUTIONS}

S.J.G., N.N., H.I.W.-N., E.M.H., R.L.Z. and A.P. performed experiments. S.J.G., R.L.Z., J.D., B.B.M. and U.B. designed experiments, analyzed, and interpreted data. S.J.G. and N.N. wrote the manuscript. S.J.G., B.B.M. and J.D. edited the manuscript.

\section{ADDITIONAL INFORMATION}

The online version of this article (https://doi.org/10.1038/s41385-018-0106-4) contains supplementary material, which is available to authorized users.

Competing interests: The authors declare no competing interests.

\section{REFERENCES}

1. Rice, T., Rubinson, L. \& Uyeki, T. Critical illness from 2009 pandemic influenza A (H1N1) virus and bacterial co-infection in the United States. Crit. Care 40, 1487-1498 (2012).

2. Peiris, J. S. M., Hui, K. P. Y. \& Yen, H.-L. Host response to influenza virus: protection versus immunopathology. Curr. Opin. Immunol. 22, 475-481 (2010).

3. Smith, A. M. \& McCullers, J. A. Secondary bacterial infections in influenza virus infection pathogenesis. Curr. Top. Microbiol. Immunol. 385, 327-356 (2014).

4. Murray, R. J. et al. Community-acquired pneumonia due to pandemic $A(H 1 N 1)$ 2009 influenzavirus and methicillin resistant Staphylococcus aureus co-infection. PLOS ONE 5(1), e8705 (2010).

5. McGill, J., Heusel, J. W. \& Legge, K. L. Innate immune control and regulation of influenza virus infections. J. Leukoc. Biol. 86, 803-812 (2009).

6. Gouwy, M. et al. Chemokines and other GPCR ligands synergize in receptormediated migration of monocyte-derived immature and mature dendritic cells. Immunobiology 219, 218-229 (2014).

7. van Helden, M. J. G., Zaiss, D. M. W. \& Sijts, A. Ja. M. CCR2 defines a distinct population of NK cells and mediates their migration during influenza virus infection in mice. PLOS ONE 7, e52027 (2012).

8. Lin, K. L., Suzuki, Y., Nakano, H., Ramsburg, E. \& Gunn, M. D. CCR2+ monocytederived dendritic cells and exudate macrophages produce influenza-induced pulmonary immune pathology and mortality. J. Immunol. 180, 2562-2572 (2008).

9. Coates B. M. et al. Inflammatory monocytes drive influenza A virus-mediated lung injury in juvenile mice. J. Immunol. 200, 2391-2404 (2018).

10. Kroetz, D. N. et al. Type I interferon induced epigenetic regulation of macrophages suppresses innate and adaptive immunity in acute respiratory viral infection. PLoS Pathog. 11, e1005338 (2015).

11. Xiong, $H$. et al. Distinct contributions of neutrophils and CCR2+ monocytes to pulmonary clearance of different klebsiella pneumoniae strains. Infect. Immun. $\mathbf{8 3}$ (9), 3418-3427 (2015).

12. Pietras, E. M. et al. A MyD88-dependent IFNyR-CCR2 signaling circuit is required for mobilization of monocytes and host defense against systemic bacterial challenge. Cell Res. 21, 1068-1079 (2011).

13. Xiong, $\mathrm{H}$. et al. Innate lymphocyte/Ly6C(hi) monocyte crosstalk promotes klebsiella pneumoniae clearance. Cell 165, 679-689 (2016).

14. Winter, C. et al. Lung-specific overexpression of CC chemokine ligand (CCL) 2 enhances the host defense to Streptococcus pneumoniae infection in mice: role of the CCL2-CCR2 axis. J. Immunol. 178, 5828-5838 (2007).

15. Frank, K. M. et al. Host response signature to Staphylococcus aureus alpha-hemolysin implicates pulmonary Th17 response. Infect. Immun. 80, 3161-3169 (2012).

16. Kudva, A. et al. Influenza A inhibits Th17-mediated host defense against bacterial pneumonia in mice. J. Immunol. 186, 1666-1674 (2011).

17. Cao, J. et al. Activation of IL-27 signalling promotes development of postinfluenza pneumococcal pneumonia. EMBO Mol. Med. 6, 120-140 (2014).

18. Cho, J. S. et al. IL-17 is essential for host defense against cutaneous Staphylococcus aureus infection in mice. J. Clin. Invest. 120, 1762-1773 (2010).

19. Bosmann, M. et al. CD11C+ alveolar macrophages are a source of IL-23 during lipopolysaccharide-induced acute lung injury. Shock 39, 447-452 (2013).

20. Iwakura, Y. \& Ishigame, H. The IL-23/IL-17 axis in inflammation. J. Clin. Invest. 116, 1218-1222 (2006).

21. Trinchieri, G. Interleukin-12 and the regulation of innate resistance and adaptive immunity. Nat. Rev. Immunol. 3, 133-146 (2003).

22. Robinson, K. M. et al. Influenza A exacerbates Staphylococcus aureus pneumonia by attenuating IL-1 $\beta$ production in mice. J. Immunol. 191, 5153-5159 (2013).

23. Podsiad, A. et al. MicroRNA-155 regulates host immune response to postviral bacterial pneumonia via IL-23/IL-17 pathway. Am. J. Physiol. Lung Cell Mol. Physiol. 310, L465-475 (2016).

24. Robinson, K. M. et al. Influenza A virus exacerbates Staphylococcus aureus pneumonia in mice by attenuating antimicrobial peptide production. J. Infect. Dis. 209, 865-875 (2014).
25. Sun, K. \& Metzger, D. W. Influenza infection suppresses NADPH oxidasedependent phagocytic bacterial clearance and enhances susceptibility to secondary methicillin-resistant Staphylococcus aureus infection. J. Immunol. 192, 3301-3307 (2014).

26. Gurczynski, S. J., Procario, M. C., O'Dwyer, D. N., Wilke, C. A. \& Moore, B. B. Loss of CCR2 signaling alters leukocyte recruitment and exacerbates gammaherpesvirus-induced pneumonitis and fibrosis following bone marrow transplantation. Am. J. Physiol. Lung Cell. Mol. Physiol. 311, L611-627 (2016).

27. Bauman, K. A. et al. The antifibrotic effects of plasminogen activation occur via prostaglandin E2 synthesis in humans and mice. J. Clin. Invest. 120(6), 1950-1960 (2010).

28. Ballinger, M. N. et al. Role of granulocyte macrophage colony-stimulating factor during gram-negative lung infection with Pseudomonas aeruginosa. Am. J. Respir. Cell Mol. Biol. 34(6), 766-774 (2006).

29. Dawson, T. C., Beck, M. A., Kuziel, W. A., Henderson, F. \& Maeda, N. Contrasting effects of CCR5 and CCR2 deficiency in the pulmonary inflammatory response to influenza A virus. Am. J. Pathol. 156, 1951-1959 (2000).

30. Ye, P. et al. Requirement of interleukin 17 receptor signaling for lung CXC chemokine and granulocyte colony-stimulating factor expression, neutrophil recruitment, and host defense. J. Exp. Med. 194(4), 519-527 (2001).

31. Chen, K. et al. IL-17 receptor signaling in the lung epithelium is required for mucosal chemokine gradients and pulmonary host defense against K. pneumoniae. Cell Host. Microbe 20(5), 596-605 (2016).

32. Maaten, L. V. D. \& Hinton, G. Visualzing data using t-SNE. J. Mach. Learn. Res. 9, 2579-2605 (2008).

33. Lin, K. L., Sweeney, S., Kang, B. D., Ramsburg, E. \& Gunn, M. D. CCR2-antagonist prophylaxis reduces pulmonary immune pathology and markedly improves survival during influenza infection. J. Immunol. 186, 508-515 (2011).

34. Beauchamp, N. M. \& Yammani, R. D. Alexander-Miller Ma. CD8 marks a subpopulation of lung-derived dendritic cells with differential responsiveness to viral infection and toll-like receptor stimulation. J. Virol. 86, 10640-10650 (2012).

35. Damjanovic, D. et al. Negative regulation of lung inflammation and immunopathology by TNF-alpha during acute influenza infection. Am. J. Pathol. 179(6), 2963-2976 (2011).

36. Sun, K. \& Metzger, D. W. Inhibition of pulmonary antibacterial defense by interferon-gamma during recovery from influenza infection. Nat. Med. 14, 558-564 (2008).

37. Huang, Y. et al. Innate and adaptive immune responses in patients with pandemic influenza A(H1N1)pdm09. Arch. Virol. 158, 2267-2272 (2013).

38. Fujimura, N. et al. CCR2 inhibition sequesters multiple subsets of leukocytes in the bone marrow. Sci. Rep. 5, 11664 (2015).

39. Souto, F. O. et al. Essential role of CCR2 in neutrophil tissue infiltration and multiple organ dysfunction in sepsis. Am. J. Respir. Crit. Care. Med. 183, 234-242 (2011).

40. Feuerstein, R., Seidl, M., Prinz, M. \& Henneke, P. MyD88 in macrophages is critical for abscess resolution in staphylococcal skin infection. J. Immunol. 194, 2735-2745 (2015).

41. Deniset, J. F., Surewaard, B. G., Lee, W. Y. \& Kubes, P. Splenic Ly6Ghigh mature and Ly6Gint immature neutrophils contribute to eradication of S. pneumoniae. J. Exp. Med. 214, 1333-1350 (2017).

42. Moltedo, B. et al. Cutting edge: stealth influenza virus replication precedes the initiation of adaptive immunity. J. Immunol. 183, 3569-3573 (2009).

43. Hagau, N. et al. Clinical aspects and cytokine response in severe H1N1 influenza $A$ virus infection. Crit. Care. 14, R203 (2010).

44. Bermejo-Martin, J. F. et al. Th1 and Th17 hypercytokinemia as early host response signature in severe pandemic influenza. Crit. Care. 13, R201 (2009).

45. Cheng, P. et al. Role of gamma-delta T cells in host response against Staphylococcus aureus-induced pneumonia. BMC Immunol. 13, 38 (2012).

46. Small, C. L. et al. NK cells play a critical protective role in host defense against acute extracellular Staphylococcus aureus bacterial infection in the lung. J. Immunol. 180, 5558-5568 (2008).

47. Mulcahy, M. E., Leech, J. M., Renauld, J. C., Mills, K. H. \& McLoughlin, R. M. Interleukin-22 regulates antimicrobial peptide expression and keratinocyte differentiation to control Staphylococcus aureus colonization of the nasal mucosa. Mucosal Immunol. 9, 1429-1441 (2016).

48. Barin, J. G. et al. Collaborative interferon-gamma and Interleukin-17 signaling protects the oral mucosa from Staphylococcus aureus. Am. J. Pathol. 186 2337-2352 (2016).

49. Kinnebrew, Ma et al. Interleukin 23 production by intestinal CD103+CD11b+ dendritic cells in response to bacterial flagellin enhances mucosal innate immune defense. Immunity 36, 276-287 (2012).

50. Helft, J. et al. Cross-presenting CD103+ dendritic cells are protected from influenza virus infection. J. Clin. Invest. 122, 4037-4047 (2012).

51. Robinson, K. M. et al. The role of IL-27 in susceptibility to post-influenza Staphylococcus aureus pneumonia. Respir. Res. 16, 10 (2015). 
CCR2 mediates increased susceptibility to post-H1N1 bacterial pneumonia...

SJ Gurczynski et al.

530

52. Wang, $\mathrm{L}$. et al. Selective depletion of $\mathrm{CD} 11 \mathrm{c}(+) \mathrm{CD} 11 \mathrm{~b}(+)$ dendritic cells partially abrogates tolerogenic effects of intravenous MOG in murine EAE. Eur. J. Immunol. 46, 2454-2466 (2016).

53. Stumhofer, J. S. et al. Interleukin 27 negatively regulates the development of interleukin 17-producing $T$ helper cells during chronic inflammation of the central nervous system. Nat. Immunol. 7, 937-945 (2006).
54. Osterholzer, J. J. et al. Accumulation of CD11b+ lung dendritic cells in response to fungal infection results from the CCR2-mediated recruitment and differentiation of Ly-6Chigh monocytes. J. Immunol. 183, 8044-8053 (2009).

55. Plantinga, $\mathrm{M}$. et al. Conventional and monocyte-derived $\mathrm{CD} 11 \mathrm{~b}(+)$ dendritic cells initiate and maintain $\mathrm{T}$ helper 2 cell-mediated immunity to house dust mite allergen. Immunity 38, 322-335 (2013). 